\title{
Unitary Representations of some Infinite Dimensional Groups
}

\author{
Graeme Segal \\ St. Catherine's College, Oxford University, Oxford OX1 3UJ, England
}

\begin{abstract}
We construct projective unitary representations of (a) $\operatorname{Map}\left(S^{1} ; G\right)$, the group of smooth maps from the circle into a compact Lie group $G$, and (b) the group of diffeomorphisms of the circle. We show that a class of representations of $\operatorname{Map}\left(S^{1} ; T\right)$, where $T$ is a maximal torus of $G$, can be extended to representations of $\operatorname{Map}\left(S^{1} ; G\right)$,
\end{abstract}

\section{Introduction}

One object of this paper is to describe a series of projective unitary representations of the group of (orientation preserving) diffeomorphisms of the circle. They are characterized, and distinguished from other known representations ([8], [13]), by the property of having "positive energy", which means that the rotation of the circle through an angle $\alpha$ is represented by $e^{-i \alpha K}$ where $K$ is a positive operator.

In their infinitesimal form, i.e. as representations of Vect $\left(S^{1}\right)$, the Lie algebra of smooth vector fields on the circle, the representations have been known for some time to physicists ([5], [3]) in connection with the quantization of strings moving relativistically. (Vect $\left(S^{1}\right)$ is called by physicists the Virasoro algebra.) I have tried to explain briefly in an appendix to this paper how the representations are relevant to the theory of strings; but as a crude oversimplification one can say that one wants to describe unparametrized strings but finds it more convenient to describe parametrized strings: the group of diffeomorphisms acts on the Hilbert space of states of a parametrized string by changing parametrization.

The infinitesimal version of the representations has also been described by $\operatorname{Kač}([7][7 \mathrm{a}])$.

My approach to the construction of the representations involves constructing irreducible representations of another family of groups. For any Lie group $G$ the group Diff $\left(S^{1}\right)$ of orientation preserving diffeomorphisms of the circle $S^{1}$ is a group of automorphisms of the group $\operatorname{Map}\left(S^{1} ; G\right)$ of smooth maps from $S^{1}$ to $G$ (under pointwise composition). Taking first $G=\mathbb{T}$, the circle group, I shall construct an irreducible projective unitary representation of $\operatorname{Map}\left(S^{1} ; \mathbb{T}\right)$ on a Hilbert space $H$. Then I shall show, what seems to me rather surprising, that any representation 
of $\operatorname{Map}\left(S^{1} ; \mathbb{T}\right)$ belonging to a certain class extends canonically to a representation of $\operatorname{Map}\left(S^{1} ; S U_{2}\right)$ on the same Hilbert space $H$, when $\mathbb{T}$ is identified with the diagonal matrices in $S U_{2}$. The next step is to show that $\operatorname{Diff}\left(S^{1}\right)$ acts projectively on $H$, intertwining with the action of $\operatorname{Map}\left(S^{1} ; S U_{2}\right)$, so that we have a projective action of the semidirect product $\operatorname{Diff}\left(S^{1}\right) \tilde{\times} \operatorname{Map}\left(S^{1} ; S U_{2}\right)$. This last group contains the product $\operatorname{Diff}\left(S^{1}\right) \times S U_{2}$, where $S U_{2}$ is identified with the constant maps $S^{1} \rightarrow S U_{2}$. We shall see that under the action of $\operatorname{Diff}\left(S^{1}\right) \times S U_{2}$ the space $H$ can be decomposed

$$
H=\underset{q=0,1,2, \ldots}{\bigoplus} P_{q^{2}} \otimes D_{q},
$$

where the $P_{q^{2}}$ are distinct irreducible representations of $\operatorname{Diff}\left(S^{1}\right)$, and $D_{q}$ is the $(2 q+1)$-dimensional irreducible representation of $S U_{2} \cdot{ }^{1}$

Defining the action of $\operatorname{Diff}\left(S^{1}\right)$ on $H$ involves constructing the metaplectic representation of a certain infinite dimensional symplectic group. This construction is due in essence to Shale [10], but has been developed more explicitly by Vergne [12]. My method is superficially, but not fundamentally, different from hers.

The theorem that a suitable projective representation of $\operatorname{Map}\left(S^{1} ; \mathbb{T}\right)$ can be extended to one of $\operatorname{Map}\left(S^{1} ; S U_{2}\right)$ can be generalized in the following way. If $G$ is a simply connected and simply laced (cf. Sect. 4) compact Lie group with a maximal torus $T$ then a class of projective representations of $\operatorname{Map}\left(S^{1} ; T\right)$ can be extended to $\operatorname{Map}\left(S^{1} ; G\right)$. Among other things this gives one a new and interesting explicit construction of the fundamental irreducible projective representations of $\operatorname{Map}\left(S^{1} ; G\right)$. On these representations too the group $\operatorname{Diff}\left(S^{1}\right)$ acts, and again they can be decomposed under $\operatorname{Diff}\left(S^{1}\right) \times G$.

Since writing the present work I have learnt that the extension theorem has been proved independently by Frenkel and Kač [0], who have also observed that the essential ingredient is the "Veneziano vertex" of the theory of strings [5].

All the representations we shall be concerned with are projective, i.e. they are really representations of central extensions of the groups in question by the circle

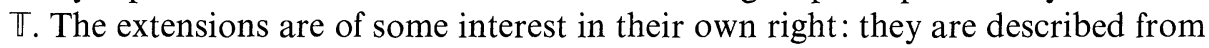
various points of view in Sect. 7 .

To get some idea of the position of the representations constructed here among the totality of representations of $\operatorname{Map}\left(S^{1} ; G\right)$ and $\operatorname{Diff}\left(S^{1}\right)$ one can consider the "orbits" in the coadjoint representation in the manner of Kirillov and Kostant. That is done in Sect. 8. In the case of $\operatorname{Map}\left(S^{1} ; G\right)$ the results are very satisfactory, in that for projective representations with the cocycle we are considering the orbit method suggests that the unitary representations constructed in this paper are the only ones which exist. These representations are precisely the ones found by Kač, whose method, like mine, constructs only representations of positive energy (i.e. "with a lowest weight"). I should perhaps mention at this point that Gel'fand and others have constructed non-projective representations of $\operatorname{Map}\left(S^{1} ; G\right)$ of a completely different type, not of positive energy (cf. [14].)

In the case of $\operatorname{Diff}\left(S^{1}\right)$ the predictions of the orbit method are not so clear. The

1 To avoid misunderstanding I should emphasize that in this paper the irreducibility of $P_{q^{2}}$ is proved only when $q=0$ (cf. Sect. 6). The irreducibility has been proved in general by Kač 
coadjoint representation of $\operatorname{Diff}\left(S^{1}\right)$ has a natural interpretation in terms of secondorder differential equations on the circle--so-called "Hill's equations" - and the orbits are classified by their monodromy. There seems to be at least a rough correspondence between the orbits and the unitary representations.

My interest in the subject of this paper was aroused by Goldstone, and all the results about the decomposition of the irreducible representations of $\operatorname{Map}\left(S^{1} ; \mathbb{T}\right)$ under the action of $\operatorname{Diff}\left(S^{1}\right)$ were told to me by him. I am most grateful for the stimulus and instruction he has given me.

The decomposition of the representation of $\operatorname{Map}\left(S^{1} ; G\right)$ in the general case was explained to me by Macdonald. I have also been greatly helped in connection with the metaplectic representation by Kazhdan and Vergne, and in understanding the action of Diff $\left(S^{1}\right)$ on Hill's equations by Hitchin.

It will be obvious that the results of this paper have mostly already been obtained, at least on the infinitesimal level, by Kač. I hope nevertheless that my methods and point of view are sufficiently different to justify their publication. My methods, on the other hand, are in some sense familiar among physicists: in connection with Sect. 5 I should mention the work of Goddard and Horsley [3], and for the construction of Sect. 4 the volume [5], passim.

\section{The Central Extension of Map $\left(S^{1} ; T\right)$}

The group $M=\operatorname{Map}\left(S^{1} ; \mathbb{T}\right)$ is disconnected, with its connected components indexed by the winding number. There is an exact sequence

$$
0 \rightarrow \mathbb{Z} \stackrel{\times 2 \pi}{\longrightarrow} \operatorname{Map}\left(S^{1} ; \mathbb{R}\right) \rightarrow \operatorname{Map}\left(S^{1} ; \mathbb{I}\right) \stackrel{w}{\longrightarrow} \mathbb{Z} \rightarrow 0,
$$

where the middle map is $f \mapsto e^{i f}$, and $w$ is the winding number. Our first task is to

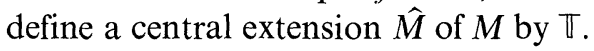

Let $F$ denote the vector space of smooth functions $f: \mathbb{R} \rightarrow \mathbb{R}$ such that

$$
\Delta_{f}=\frac{1}{2 \pi}(f(\theta+2 \pi)-f(\theta))
$$

is constant. The subgroup of $F$ consisting of $f$ such that $\Delta_{f} \in \mathbb{Z}$ will be denoted by $F_{\mathbb{Z}}$. Any element of $M$ can be written $e^{i f}$, with $f \in F_{\mathbb{Z}}$, when $S^{1}$ is thought of as $\mathbb{R} / 2 \pi \mathbb{Z}$.

There is a skew bilinear form $S: F \times F \rightarrow \mathbb{R}$ defined by

$$
S(f, g)=\frac{1}{4 \pi} \int_{0}^{2 \pi}\left(f^{\prime}(\theta) g(\theta)-f(\theta) g^{\prime}(\theta)\right) d \theta+\frac{1}{4 \pi}(f(2 \pi) g(0)-f(0) g(2 \pi)) .
$$

(Observe that $S(f, 1)=\Delta_{f}$.) We define the group $\hat{M}$ as the space $\mathbb{T} \times M$ with the composition law

$$
\left(\lambda, e^{i f}\right) \cdot\left(\mu, e^{i g}\right)=\left(\lambda \mu e^{-i S(f, g)}, e^{i(f+g)}\right) .
$$

It is fundamental for our purposes that the group $\operatorname{Diff}\left(S^{1}\right)$ acts on $\hat{M}$ as a group of automorphisms. That is true because the cocycle $c: M \times M \rightarrow \mathbb{T}$ given by $c\left(e^{i f}, e^{i g}\right)=e^{-i S(f, g)}$ which defines $\hat{M}$ is invariant under $\operatorname{Diff}\left(S^{1}\right)$. 
It is not hard to determine all the central extensions of $M$ by $\mathbb{T}$ which are invariant under $\operatorname{Diff}\left(S^{1}\right)$. Indeed any such extension $\tilde{M}$ can be identified with $\mathbb{T} \times M$ as a space. Let $C$ denote the circle of constant function in $M$, and let $\varepsilon$ denote any element of $M$ of winding number 1 . Then $c \mapsto \varepsilon c \varepsilon^{-1} c^{-1}$, where the multiplication is in $\hat{M}$, is a map $C \rightarrow \mathbb{T}$.

Proposition (2.1) The central extensions $\mathbb{T} \rightarrow \tilde{M} \rightarrow M$ which are invariant under $\operatorname{Diff}\left(S^{1}\right)$ are completely classified by the winding number of the map $C \rightarrow \mathbb{T}$ just described. Any integral winding number can occur.

I shall omit the proof. The extension we are denoting by $\hat{M}$ has winding number 2. To obtain an extension with winding number 1 one can replace the skew form $S$ used to define $M$ with the (non-skew) bilinear form $s: F \times F \rightarrow \mathbb{R}$ defined by

$$
s(f, g)=\frac{1}{4 \pi} \int_{0}^{2 \pi} f^{\prime}(\theta) g(\theta) d \theta+\frac{1}{2} \Delta_{f} g(0) .
$$

(Notice that $s(f, g)-s(g, f)=S(f, g) \cdot)$ The form $s$ is not invariant under $\operatorname{Diff}\left(S^{1}\right)$, but nevertheless a double covering of $\operatorname{Diff}\left(S^{1}\right)$ acts on the associated extension $\tilde{M}$ by

$$
\phi^{*}(\lambda, \gamma)=\left(\lambda\left\{\gamma(\phi(0)) \gamma(0)^{-1}\right\}^{-(1 / 2) w(\gamma)}, \phi^{*} \gamma\right)
$$

\section{The Projective Representation of Map $\left(S^{1} ; T\right)$}

We shall construct a unitary representation of $\hat{M}$ on a Hilbert space $H$. It is more convenient notationally, however, to describe it as a projective representation of $M$, i.e. to associate to each $\gamma$ in $M$ an operator $T(\gamma): H \rightarrow H$ such that

$$
T(\gamma) T\left(\gamma^{\prime}\right)=c\left(\gamma, \gamma^{\prime}\right) T\left(\gamma \gamma^{\prime}\right)
$$

where $c: M \times M \rightarrow \mathbb{T}$ is the cocycle described in the previous section.

We begin with the identity component $M_{0}$ of $M$. If $C \subset M_{0}$ is the constants, then $M_{0} / C$ can be identified with the vector space $V=\operatorname{Map}\left(S^{1} ; \mathbb{R}\right) /($ constants). We shall construct a projective unitary representation of $V$ on a Hilbert space $H_{0}$; then, regarding $H_{0}$ as a representation of $M_{0}$, we shall define $H$ as the representation of $M$ induced from $H_{0}$.

The skew form $S$ induces a skew form $S: V \times V \rightarrow \mathbb{R}$. To represent the Lie algebra of the desired central extension of $V$ is to associate linearly to each $f \in V$ an operator $A(f)$ so that

$$
[A(f), A(g)]=2 i S(f, g) \text {. }
$$

(Then the group element $e^{i f}$ in $M_{0}$ will be represented by $e^{i A(f)}$.) That is, we must represent the "canonical commutation relations" associated to $V$ and $S$. We do this using the standard representation on Fock space.

A complex polarization of $V$ for the form $S$ means a decomposition $V_{\mathbb{C}}=W \oplus \bar{W}$, where $V_{\mathbb{C}}$ is the complexification of $V$, such that $S$ is identically zero on $W .(\bar{W}$ denotes the complex conjugate of $W$.) Then

$$
\left(w_{1}, w_{2}\right) \mapsto\left\langle w_{1}, w_{2}\right\rangle=2 i S\left(\bar{w}_{1}, w_{2}\right)
$$


is a hermitian form on $W$. If this is positive definite, making $W$ a pre-Hilbert-space, we shall call $W$ a positive polarization.

In our case, where $V=\operatorname{Map}\left(S^{1} ; \mathbb{R}\right) /($ constants $)$, there is a canonical positive polarization, in which $W$ is the space of smooth maps $f: S^{1} \rightarrow \mathbb{C}$ which extend to holomorphic functions on the disk $D=\{z \in \mathbb{C}:|z| \leqq 1\}$ (modulo constants). The spaces $W$ and $\bar{W}$ are isotropic for $S$ by Cauchy's theorem, and the form $\langle$,$\rangle is$ positive-definite. In fact if $f(z)=\sum_{n>0} a_{n} z^{n}$ and $g(z)=\sum_{n>0} b_{n} z^{n}$ then

$$
\begin{aligned}
\langle f, g\rangle & =\sum 2 n \bar{a}_{n} b_{n} \\
& =\frac{2}{\pi} \int_{D} \overline{f^{\prime}(z)} g^{\prime}(z) d \mu(z) \\
& =\frac{1}{\pi i} \int_{D} \overline{d f} \wedge d g
\end{aligned}
$$

where $\mu$ is Lebesgue measure on $D$.

Let us consider the symmetric algebra $S(W)$ of $W$. The elements of $W$ act on this by multiplication: we write $A(w): S^{k}(W) \rightarrow S^{k+1}(W)$ for multiplication by $w$. For each $\bar{w}$ in $\bar{W}$ there is a unique derivation $A(\bar{w}): S^{k}(W) \rightarrow S^{k-1}(W)$ of the algebra $S(W)$ whose action on $S^{1}(W)=W$ is given by

$$
A(\bar{w}) \cdot u=\langle w, u\rangle \text {. }
$$

For any $f$ in $V$ we define $A(f): S(W) \rightarrow S(W)$ by

$$
A(f)=A\left(f_{+}\right)+A\left(f_{-}\right)
$$

where $f=f_{+}+f_{-}$with $f_{+} \in W$ and $f_{-} \in \bar{W}$. The relation

$$
[A(f), A(g)]=2 i S(f, g)
$$

follows at once from the fact that $A\left(f_{-}\right)$and $A\left(g_{-}\right)$are derivations.

The inner product $<,>$ on $W$ extends to $S(W)$ by

$$
\left\langle w_{1} w_{2} \ldots w_{k}, w_{1} w_{2} \ldots w_{k}\right\rangle=\sum\left\langle w_{i_{1}}, w_{1}\right\rangle\left\langle w_{i_{2}}, w_{2}\right\rangle \ldots\left\langle w_{i_{k}}, w_{k}\right\rangle,
$$

where the sum is over all permutations $\left(i_{1}, \ldots, i_{k}\right)$ of $(1, \ldots, k)$. With respect to this inner product the operators $A(w)$ and $A(\bar{w})$ are adjoint for any $w$ in $W$, and so $A(f)$ is self-adjoint for real $f$ in $V$.

We define $H_{0}$ as the Hilbert space completion $\hat{S}(W)$ of $S(W)$. The $A(f)$ for $f$ in $V$ can be thought of as unbounded operators in $H_{0}$; we have to show that they are self-adjoint in the appropriate sense so that they define unitary operators $e^{i A(f)}$ in $H_{0}$.

It is easy to check that the following estimates hold:

$$
\begin{aligned}
& \|A(w) \cdot \xi\| \leqq \sqrt{k+1} \cdot\|w\|\|\xi\|, \\
& \|A(\bar{w}) \cdot \xi\| \leqq \sqrt{k} \cdot\|w\|\|\xi\|,
\end{aligned}
$$

when $w \in W$ and $\xi \in S^{k}(W)$. From these we deduce

$$
\|A(f) \cdot \xi\| \leqq 2 \sqrt{k+1} .\|f\|\|\xi\|
$$


when $f \in V$ and $\xi \in S^{0}(W) \oplus \ldots \oplus S^{k}(W)$, and $\|f\|$ is defined as $\left\|f_{+}\right\|$. It follows that the series

$$
\sum \frac{1}{k !}(i A(f))^{k} \cdot \xi
$$

converges in $H_{0}$ for each $\xi \in S(W)$, and defines

$$
e^{i A(f)}: S(W) \rightarrow H_{0}
$$

But if $\xi, \eta \in S(W)$ then

$$
\begin{aligned}
\left\langle e^{i A(f)} \xi, e^{i A(f)} \eta\right\rangle & =\sum_{k, m}\left\langle\xi, \frac{(-i A(f))^{k}(i A(f))^{m}}{k !} \eta\right\rangle \\
& =\langle\xi, \eta\rangle,
\end{aligned}
$$

because $\sum_{k}(-1)^{k} / k ! m !=0$ when $n>0$.

So $e^{\substack{k+m=n \\ A(f)}}$ extends to a unitary operator $H_{0} \rightarrow H_{0}$. In view of the identity $e^{P} e^{Q}=$ $e^{(1 / 2)[P, Q]} e^{P+Q}$, which holds whenever $[\mathrm{P}, \mathrm{Q}]$ commutes with $P$ and $Q$, we find

$$
e^{i A(f)} e^{i A(g)}=e^{-i S(f, g)} e^{i A(f+g)},
$$

which shows that $T\left(e^{i f}\right)=e^{i A(f)}$ defines the desired projective unitary representation of the group $V=M_{0} / C$ on $H_{0}$.

Let us regard $M_{0}$ as $V \times C$ by identifying $V$ with the subspace $\left\{f: \int f=0\right\}$ of $\operatorname{Map}\left(S^{1} ; \mathbb{R}\right)$. Then for each integer $k$ there is a representation $H_{k}$ of $M_{0}$ which coincides with $H_{0}$ as a representation of $V$, but in which $\lambda \in C$ acts as $\lambda^{k}$. The projective representation of $M$ induced from $H_{0}$ can be best be described as the Hilbert space direct sum $H=\bigoplus_{k \in \mathbb{Z}} H_{2 k}$, on which the canonical element $e^{i n \theta}$ of $M$ of winding number $n$ acts as the "identity" map $H_{2 k} \rightarrow H_{2(k+n)}$. The "vacuum vector" in $H_{2 k}$ will be denoted by $\Omega_{2 k}$, and $H_{2 k}$ will be thought of as $\hat{S}(W) \cdot \Omega_{2 k}$.

To conclude this section I shall show that the representation of $\hat{M}$ we have constructed is irreducible, and is essentially the only representation of its kind.

We shall see in Sect. 5 that $\operatorname{Diff}\left(S^{1}\right)$ acts (projectively) on $H$ intertwining with the representation of $\hat{M}$. But it is meanwhile easy to see that the subgroup $R$ of rigid rotations of the circle does so. For $R$ acts naturally on $W$, and hence on $\hat{S}(W)=H_{0}$. To fix its action on the other $H_{2 k}$ it is enough to see how it acts on the vacuum vectors $\Omega_{2 k}$. We have no choice in prescribing this, for if $r_{\alpha}$ is the rotation through $\alpha$, and $\varepsilon_{k}$ is the function $e^{i k \theta}$ in $M$, then we must have

$$
\begin{aligned}
r_{\alpha} \Omega_{2 k} & =r_{\alpha} T\left(\varepsilon_{k}\right) \Omega_{0}=r_{\alpha} T\left(\varepsilon_{k}\right) r_{\alpha}^{-1} \Omega_{0} \\
& =T\left(r_{\alpha} \cdot \varepsilon_{k}\right) \Omega_{0}=T\left(r_{\alpha} \cdot \varepsilon_{k}\right) T\left(\varepsilon_{-k}\right) \Omega_{2 k} \\
& =c\left(r_{\alpha} \cdot \varepsilon_{k}, \varepsilon_{-k}\right) T\left(e^{i k \alpha}\right) \Omega_{2 k}=e^{i k^{2} \alpha} \cdot e^{-2 i k^{2} \alpha} \cdot \Omega_{2 k} \\
& =e^{-i k^{2} \alpha} \Omega_{2 k} .
\end{aligned}
$$

On the other hand there is no difficulty in seeing that this action on the $\Omega_{2 k}$ does define an action of $R$ on $H$ which intertwines with $M$.

Let $H(q)$ denote the part of $H$ where $r_{\alpha}$ acts as $e^{-i q \alpha} . H(q)$ is finite dimensional 
and vanishes when $q<0$. (Its dimension is the coefficient of $x^{q}$ in $\left.\prod_{n>0}\left(1-x^{n}\right)^{-1} \cdot \sum_{m \in Z} x^{m^{2}} \cdot\right)$

I shall call a representation of $M$ symmetric if its isomorphism class does not change when twisted by a rotation of the circle. If the intertwining operator corresponding to the rotation $r_{\alpha}$ is $e^{-i \alpha K}$ where $K$ is a positive operator I shall say the representation has positive energy. ( $K$ is determined only up to the addition of an integral of $2 \pi$, so it would be better to require the spectrum of $K$ to be bounded below. But the ambiguity is removed when one extends the action of $R$ to $\operatorname{Diff}\left(S^{1}\right)$ ).

Proposition (3.1) The projective representation of $M$ on $H$ is irreducible, symmetric, and of positive energy. It is one of precisely two such representations of $M$ with the given cocycle $c$.

Proof. First consider the action of $M_{0}$ on $H_{0}=\hat{S}(W)$. Clearly the vacuum vector $\Omega_{0}$ is cyclic. Any possible decomposition $H_{0}=H_{0}^{\prime} \oplus H_{0}^{\prime \prime}$ must respect the grading by the eigenspaces $H_{0}(q)=H_{0} \cap H(q)$, so $\Omega_{0}$ must belong to either $H_{0}^{\prime}$ or $H_{0}^{\prime \prime}$. Thus $H_{0}$ is irreducible under $M_{0}$. It follows that all $H_{k}$ are irreducible under $M_{0}$, and hence that $H$ is irreducible under $M$.

But the $H_{k}$ are the only irreducible representations of $M_{0}$ of positive energy. To see that, it is enough (since $M_{0}=C \times V$ ) to show that $\hat{S}(W)$ is the only irreducible representation of $V$ of positive energy. But any such representation would have to contain a vector $\Omega_{0}$ annihilated by $A(\bar{w})$ for $\bar{w} \in \bar{W}$, for $A(\bar{w})$ lowers energy. On the other hand $\hat{S}(W)$ is freely generated by the action of the $A(f)$, for $f \in V$, subject to this constraint; so it is the only possible representation.

Finally, the group $\hat{M}$ is a semidirect product $\mathbb{Z} \tilde{\times} M_{0}$, and the representations $H_{k}$ and $H_{m}$ of $\hat{M}_{0}$ are conjugate under $\hat{M}$ if and only if $k \equiv m(\bmod 2)$. So by Mackey's theorem there are two irreducible positive-energy representations of $\hat{M}$ which are faithful on the centre, restricting respectively to $\oplus H_{2 k}$ and $\oplus H_{2 k+1}$ as representations of $\hat{M}_{0}$.

\section{The action of $\operatorname{Map}\left(S^{1} ; S U_{2}\right)$}

The group $M=\operatorname{Map}\left(S^{1} ; \mathbb{T}\right)$ can be thought of as a subgroup of $G=\operatorname{Map}\left(S^{1} ; S U_{2}\right)$

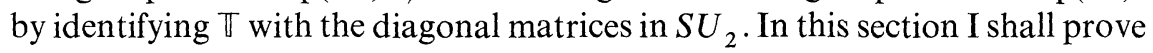

Proposition (4.1) . Let $H$ be a projective unitary representation of $M$ with the multiplier $c$. Suppose that $H$ is symmetric with positive energy, and that each eigenspace $H(q)$ of the rotations of the circle is finite-demensional. Then the action of $M$ on $H$ extends canonically to a projective unitary representation of $G$.

To begin with we shall define the representation on the Lie algebra of $G$. In fact we shall consider first the smaller Lie algebra $\operatorname{Map}_{\text {alg }}\left(S^{1} ; s u_{2}\right)$ of all algebraic maps $f: S^{1} \rightarrow s u_{2}$, i.e. those of the form

$$
f(\theta)=\sum_{k=-N}^{N} L_{k} e^{i k \theta},
$$

where the $L_{k}$ are complex $2 \times 2$ matrices. 
As we shall be considering unbounded operators it is useful to introduce a "rigging" (cf. [1] (Chap. 1, Sect. 4)) of the Hilbert space $H$. Let $\breve{H}$ denote the direct sum and $\hat{H}$ the direct product of the $H(q)$. Then $\breve{H} \subset H \subset \hat{H}$, and $\breve{H}$ and $\hat{H}$ are dual to each other.

For any $\alpha \in S^{1}$ and $0 \leqq \lambda<1$ let us consider the element $\gamma_{\alpha, \lambda}: S^{1} \rightarrow \mathbb{T}$ of $M$ defined by

$$
\gamma_{\alpha, \lambda}(\theta)=\frac{\lambda-e^{i(\theta-\alpha)}}{1-\lambda e^{i(\theta-\alpha)}}
$$

This should be thought of as a "blip" situated at the point $\alpha$ of the circle, becoming sharper as $\lambda \rightarrow 1$. It has winding number 1 , and if $\lambda$ is close to 1 then $\gamma_{\alpha, \lambda}(\theta)$ is close to 1 except in a small neighbourhood of $\theta=\alpha$, of length roughly $1-\lambda$, where it winds once around $\mathbb{T}$. In fact $\gamma_{\alpha, \lambda}(\theta)=e^{i f_{\alpha, \lambda}(\theta)}$, where $f_{\alpha, \lambda}(\theta)$ is the angle $A P A^{\prime}$ in the diagram, where $P=e^{i \theta}, A=\lambda e^{i \alpha}$, and $A^{\prime}=\lambda^{-1} e^{i \alpha}$.

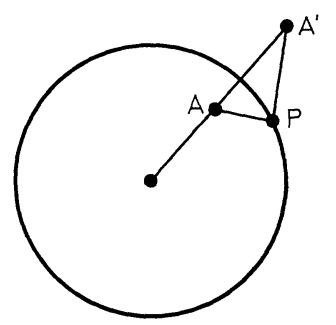

Fig, 1

To $\gamma_{\alpha, \lambda} \in M$ corresponds a unitary operator $T\left(\gamma_{\alpha, \lambda}\right)$ on $H$. I shall prove that as $\lambda \rightarrow 1$ the operator $\left(1-\lambda^{2}\right)^{-1} T\left(\gamma_{\alpha, \lambda}\right)$ tends in a certain sense to a definite but highly singular operator $B_{\alpha}{ }^{2}$

We can write $f_{\alpha, \lambda}=q_{\alpha}+f_{\alpha, \lambda}^{+}+f_{\alpha, \lambda}^{-}$, where

$$
\begin{aligned}
& f_{\alpha, \lambda}^{+}(\theta)=i \log \left(1-\lambda e^{i(\theta-\alpha)}\right)=-i \sum_{n>0} \frac{\lambda^{n}}{n} e^{i n(\theta-\alpha)}, \\
& f_{\alpha, \lambda}^{-}(\theta)=-i \log \left(1-\lambda e^{-i(\theta-\alpha)}\right), \text { and } \\
& q_{\alpha}(\theta)=\pi+\theta-\alpha .
\end{aligned}
$$

Then $f_{\alpha, \lambda}^{+} \in W$ and $f_{\alpha, \lambda}^{-} \in \bar{W}$. Furthermore

so that

$$
\begin{aligned}
S\left(f_{\alpha, \lambda}^{+}, f_{\alpha, \lambda}^{-}\right) & =-\frac{1}{2 \pi} \int f_{\alpha, \lambda}^{+} d f_{\alpha, \lambda}^{-} \\
& =-\frac{1}{2 \pi} \int \frac{\log (1-\lambda z)}{1-\lambda z^{-1}} \cdot \lambda z^{-2} d z \\
& =-i \log \left(1-\lambda^{2}\right)
\end{aligned}
$$

$$
T\left(\gamma_{\alpha, \lambda}\right)=\left(1-\lambda^{2}\right) T\left(e^{i q_{\alpha}}\right) e^{i A_{\alpha}^{+}, \lambda} e^{i A_{\alpha, \lambda}}
$$

where $A_{\alpha, \lambda}^{ \pm}=A\left(f_{\alpha, \lambda}^{ \pm}\right)$.

2 In the language of quantum field theory $B_{\alpha}$ is, as a function of $\alpha$, an "operator-valued distribution" 
The operators $e^{i A_{\tilde{\alpha}, \lambda}^{+}}$and $e^{i A_{\tilde{\alpha}, \lambda}}$ can be expanded

$$
\begin{aligned}
& e^{i A_{\alpha, \lambda}^{+} \lambda}=\sum_{n \geqq 0} \lambda^{n} e^{-i n \alpha} C_{n}, \\
& e^{i A_{\bar{\alpha}, \lambda}}=\sum_{n \geqq 0} \lambda^{n} e^{i n \alpha} D_{n} .
\end{aligned}
$$

Here $C_{n}$ and $D_{n}$ are operators independent of $\lambda$ and $\alpha$ which map $H(q)$ into $H(\dot{q}+n)$ and $H(q-n)$ respectively.

As $H(q-n)$ is zero when $n>q$ it follows that

$$
e^{i A_{\alpha, \lambda}^{ \pm},} e^{i A_{\bar{\alpha}, \lambda}}=\sum_{m, n \geqq 0} \lambda^{n+m} e^{i(n-m) \alpha} C_{m} D_{n}
$$

tends to a well-defined operator $\check{H} \rightarrow \hat{H}$ as $\lambda \rightarrow 1$. Now $T\left(e^{i q_{\alpha}}\right)$ maps $H_{2 k}$ into $H_{2 k+2}$, and takes $H_{2 k}(q)$ into $H_{2 k+2}(q+2 k+1)$. On $H_{2 k}$ we have $T\left(e^{i q_{\alpha}}\right)=$ $e^{-(2 k+1) i \alpha} T_{0}$, where $T_{0}=T\left(e^{i q_{0}}\right)$. So

$$
B_{\alpha}=\lim _{\lambda \rightarrow 1}\left(1-\lambda^{2}\right)^{-1} T\left(\gamma_{\alpha, \lambda}\right)=\sum_{n, m \geqq 0} e^{i(n-m-2 k-1) \alpha} T_{0} C_{m} D_{n}
$$

on $H_{2 k}$.

If $\phi: S^{1} \rightarrow \mathbb{C}$ is a trigonometric polynomial, i.e. $\phi(\theta)=\sum_{r=-N}^{N} \phi_{r} e^{i r \theta}$, let

$$
B(\phi)=\frac{1}{2 \pi} \int_{0}^{2 \pi} \phi(\alpha) B_{\alpha} d \alpha
$$

Then $B(\phi)=\Sigma \phi_{m-n+2 k+1} T_{0} C_{m} D_{n}$ on $\check{H}_{2 k}$, and so it maps $\breve{H}$ into itself.

The adjoint operator $B_{\alpha}^{*}$ of $B_{\alpha}$ is given by

$$
B_{\alpha}^{*}=\lim _{\lambda \rightarrow 1}\left(1-\lambda^{2}\right)^{-1} T\left(\gamma_{\alpha, \lambda}^{-1}\right)=\sum e^{-i(n-m-2 k+1)} T_{0}^{-1} \tilde{C}_{m} \tilde{D}_{n}
$$

on $\check{H}_{2 k}$, where $\tilde{C}_{m}=D_{m}^{*}$ and $\tilde{D}_{n}=C_{n}^{*}$. We define

$$
B^{*}(\phi)=\frac{1}{2 \pi} \int_{0}^{2 \pi} \phi(\alpha) B_{\alpha}^{*} d \alpha,
$$

so that $B(\phi)^{*}=B^{*}(\bar{\phi})$.

For a trigonometric polynomial $\phi$ the operator $A(\phi)$ can be written $A(\phi)=$ $\sum \phi_{k} a_{k}$, where $a_{k}=A\left(e^{i k \theta}\right)$ maps $H(q)$ into $H(q+k)$ and hence $\breve{H}$ into itself. Furthermore for any distribution $\phi$ on $S^{1}$ one has an operator $A(\phi): \breve{H} \rightarrow \hat{H}$.

We shall now show that the operators $A(\phi), B(\phi), B^{*}(\phi)$ define a projective representation of the complex Lie algebra of algebraic maps $S^{1} \rightarrow s l_{2}(\mathbb{C})$ on the vector space $\breve{H}$. More precisely, we have

Proposition (4.2) (i) $[A(\phi), B(\psi)]=2 B(\phi \psi)$,

$$
\begin{aligned}
& {\left[A(\phi), B^{*}(\psi)\right]=-2 B^{*}(\phi \psi),} \\
& {\left[B(\phi), B^{*}(\psi)\right]=A(\phi \psi)+i S(\phi, \psi),}
\end{aligned}
$$$$
[B(\phi), B(\psi)]=\left[B^{*}(\phi), B^{*}(\psi)\right]=0 \text {. }
$$

These are the commutation relations for a projective representation 
of $\operatorname{Map}_{\mathrm{alg}}\left(S^{1} ; s l_{2}(\mathbb{C})\right)$ under the correspondence

$$
A(\phi) \leftrightarrow\left(\begin{array}{rr}
\phi & 0 \\
0 & -\phi
\end{array}\right) \quad B(\phi) \leftrightarrow\left(\begin{array}{ll}
0 & \phi \\
0 & 0
\end{array}\right), \quad B^{*}(\phi) \leftrightarrow\left(\begin{array}{ll}
0 & 0 \\
\phi & 0
\end{array}\right) .
$$

The cocycle giving the projective multiplier is

$$
(f, g) \mapsto \omega(f, g)=\frac{1}{2 \pi} \int_{0}^{2 \pi}\left\langle f^{\prime}(\theta), g(\theta)\right\rangle d \theta,
$$

where $f, g: S^{1} \rightarrow \operatorname{sl}_{2}(\mathbb{C})$, and $\langle$,$\rangle is the invariant form (A, B) \mapsto-\operatorname{trace}(A B)$ on $\mathrm{Sl}_{2}(\mathbb{C})$.

It is easy to check that in the representation the elements of $\operatorname{Map}_{\mathrm{alg}}\left(S^{1} ; s u_{2}\right)$ act on $\check{H}$ by skew operators.

Proof of Proposition (4.2). (i) It suffices to show that $\left[A(\phi), B_{\alpha}\right]=2 \phi(\alpha) B_{\alpha}$. Now $B_{\alpha}=\lim B_{\alpha}^{\lambda}$, where $B_{\alpha}^{\lambda}=\left(1-\lambda^{2}\right)^{-1} e^{i A(f \alpha, \lambda)}$. As the commutator of $A(\phi)$ and $A\left(f_{\alpha, \lambda}\right)$ is scalar we have

$$
\left[A(\phi), B_{\alpha}^{\lambda}\right]=i\left[A(\phi), A\left(f_{\alpha, \lambda}\right)\right] B_{\alpha}^{\lambda}=-2 S\left(\phi, f_{\alpha, \lambda}\right) B_{\alpha}^{\lambda} .
$$

But, regarded as a distribution, $f_{\alpha, \lambda} \rightarrow 2 \pi H_{\alpha}$ as $\lambda \rightarrow 1$, where $H_{\alpha}$ is the Heaviside function defined by $H_{\alpha}(\theta)=1$ if $\theta \geqq \alpha$, and $H_{\alpha}(\theta)=0$ if $\theta<\alpha$. So $S\left(\phi, f_{\alpha, \lambda}\right) \rightarrow 2 \pi S\left(\phi, H_{\alpha}\right)=-\phi(\alpha)$, as desired.

(ii) This is simply the adjoint of (i).

(iii) We first calculate

$$
\begin{aligned}
& {\left[B_{\alpha}^{\lambda},\left(B_{\beta}^{\lambda}\right)^{*}\right]=e^{i A_{\alpha}^{+}, \lambda} e^{i A_{\alpha, \lambda}^{-}} e^{-i A_{\beta}^{+}, \lambda} e^{i A_{\beta}, \lambda} T\left(e^{i q_{\alpha}}\right) T\left(e^{-i q_{\beta}}\right)} \\
& \text { - (similar expression) } \\
& =\left\{e^{\left[A_{\alpha, \lambda}^{-}, A_{\beta}^{+}, \lambda\right]+i(\alpha-\beta)}-e^{\left[A_{\bar{\beta}, \lambda}^{-}, A_{\alpha, \lambda}^{+}\right]-i(\alpha-\beta)}\right\} e^{i\left(A_{\alpha, \lambda}^{+}-A_{\lambda, \lambda}^{+}\right)} e^{i\left(A_{\alpha, \lambda}^{-}-A_{\bar{\beta}}^{-}, \lambda\right)} T\left(e^{-i(\alpha-\beta)}\right) .
\end{aligned}
$$

The expression in braces is

$$
\begin{gathered}
\frac{e^{i(\alpha-\beta)}}{\left(1-\lambda^{2} e^{i(\alpha-\beta)}\right)^{2}}-\frac{e^{-i(\alpha-\beta)}}{\left(1-\lambda^{2} e^{-i(\alpha-\beta)}\right)^{2}} \\
=\frac{2 i\left(1-\lambda^{4}\right) \sin (\alpha-\beta)}{\left(1-2 \lambda^{2} \cos (\alpha-\beta)+\lambda^{4}\right)^{2}} \\
=\frac{i}{\lambda 2} \frac{d}{d \alpha} \frac{1-\lambda^{4}}{1-2 \lambda^{2} \cos (\alpha-\beta)+\lambda^{4}}
\end{gathered}
$$

This tends to $-2 \pi i \delta^{\prime}(\alpha-\beta)$ as $\lambda \rightarrow 1$.

Furthermore as $\lambda \rightarrow 1$ we have

$$
\begin{aligned}
& A_{\alpha, \lambda}^{+} \rightarrow A_{\alpha}^{+}=A\left(-i \sum_{n>0} e^{i n(\theta-\alpha)}\right), \text { and } \\
& A_{\alpha, \lambda}^{-} \rightarrow A_{\alpha}^{-}=A\left(+i \sum_{n>0} e^{-i n(\theta-\alpha)}\right),
\end{aligned}
$$

and the expression

$$
e^{i\left(A_{\alpha}^{+}-A_{\beta}^{+}\right)} e^{i\left(A_{\alpha}^{-}-A_{\beta}^{-}\right)}=\sum_{p, q, r, s} e^{i(r-p) \alpha+i(s-q) \beta} C_{p} \widetilde{C}_{q} D_{r} \tilde{D}_{s}
$$

is a well-defined operator $\check{H} \rightarrow \hat{H}$. 
Thus we find

$$
\begin{aligned}
\lim _{\lambda \rightarrow 1}\left[B_{\alpha}^{\lambda},\left(B_{\beta}^{\lambda}\right)^{*}\right] & =-\pi i \delta^{\prime}(\alpha-\beta) e^{i\left(A_{\alpha}^{+}-A_{\beta}^{+}\right)} e^{i\left(A_{\alpha}^{-}-A_{\beta}^{-}\right)} T\left(e^{-i(\alpha-\beta)}\right) \\
& =-2 \pi i \delta^{\prime}(\alpha-\beta)=2 \pi \delta(\alpha-\beta) \frac{d}{d \alpha}\left(A_{\alpha}^{+}+A_{\alpha}^{-}-\alpha a_{0}\right)
\end{aligned}
$$

where we have used the relation

$$
\delta^{\prime}(x) F(x)=\delta^{\prime}(x) F(0)-\delta(x) F^{\prime}(0),
$$

and also that $T\left(e^{-i(\alpha-\beta)}\right)=e^{-i(\alpha-\beta) a_{0}}$.

$$
\text { But } A_{\alpha}^{+}+A_{\alpha}^{-}-\alpha a_{0}=A\left(2 \pi H_{\alpha}-\theta-\theta\right) \text {, so } \frac{d}{d \alpha}\left(A_{\alpha}^{+}+A_{\alpha}^{-}-\alpha a_{0}\right)=-2 \pi A\left(\delta_{\alpha}\right) \text {, }
$$

where $\delta_{\alpha}$ is the delta-function at $\alpha$. Hence

$$
\left[B_{\alpha}, B_{\beta}^{*}\right]=-2 \pi i \delta^{\prime}(a-\beta)+4 \pi^{2} \delta(\alpha-\beta) A\left(\delta_{\alpha}\right),
$$

and accordingly

$$
\left[B(\phi), B^{*}(\psi)\right]=i S(\phi, \psi)+A(\phi \psi)
$$

as we want.

(iv) The proof of this is precisely similar to that of (iii), but easier, because when we calculate $\left[B_{\alpha}^{\lambda}, B_{\beta}^{\lambda}\right]$ the analogue of the expression in braces above is

$$
\begin{aligned}
& \left(1-\lambda^{2} e^{i(\alpha-\beta)}\right)^{2} e^{-i(\alpha-\beta)}-\left(1-\lambda^{2} e^{-i(\alpha-\beta)}\right)^{2} e^{i(\alpha-\beta)} \\
& \quad=-2 i\left(1-\lambda^{4}\right) \sin (\alpha-\beta),
\end{aligned}
$$

which tends to zero as $\lambda \rightarrow 1$.

That completes the proof of (4.2).

Let us now write $P(\phi)=B(\phi)+B^{*}(\phi)$ and $Q(\phi)=i\left(B(\phi)-B^{*}(\phi)\right)$, so that $\{i A(\phi), i P(\phi), i Q(\phi)\}$ correspond respectively to the standard generators

$$
\left(\begin{array}{rr}
i \phi & 0 \\
0 & -i \phi
\end{array}\right),\left(\begin{array}{rr}
0 & i \phi \\
i \phi & 0
\end{array}\right),\left(\begin{array}{rr}
0 & -\phi \\
\phi & 0
\end{array}\right)
$$

of $\operatorname{Map}\left(S^{1} ; s u_{2}\right)$. The operators $\{i A(1), i P(1), i Q(1)\}$ define an action of the algebra $\mathrm{Su}_{2}$ on $\breve{H}$ which preserves each finite dimensional subspace $H(q)$. This action can be exponentiated to give a continuous action of the group $S U_{2}$ on $H$. We shall derive a number of consequences of this.

Let $\breve{H}$ denote the subspace of $H$ consisting of sums $\sum \xi_{q}$, with $\xi_{q} \in H(q)$, such that $\left\{\xi_{q}\right\}$ is rapidly decreasing, i.e. such that $(1+q)^{n}\left\|\xi_{q}\right\|$ is bounded as $q \rightarrow \infty$ for each $n$. $\breve{H}$ is precisely the space of vectors $\xi \in H$ such that $\theta \mapsto r_{\theta} \xi$ is a smooth function $S^{1} \rightarrow H$. The dual of $\breve{H}$ is the subspace $\hat{H}$ of $\breve{H}=\prod H(q)$ consisting of series $\sum \xi_{q}$ such that $\left\{\xi_{q}\right\}$ has polynomial growth, i.e. such that $(1+q)^{-n}\left\|\xi_{q}\right\|$ is bounded as $q \rightarrow \infty$ for some $n$. In other words $\xi \in \hat{H}$ if $\theta \mapsto r_{\theta} \xi$ is a distribution on $S^{1}$.

When $\phi=\sum \phi_{k} e^{i k \theta}$ is a smooth function on $S^{1}$ the operator $A(\phi)=\sum \phi_{k} a_{k}$ maps $\breve{H}$ into $\breve{H}$, and when $\phi$ is a distribution we find $A(\phi): \breve{H} \rightarrow \hat{H}$. But the group $\mathrm{SU}_{2}$ acts on $H$, and by conjugating with appropriate elements of it, one can transform the operators $P(\phi)$ and $Q(\phi)$, which are defined when $\phi$ is a trigonometric 
polynomial, into $A(\phi)$. It follows not only that $P(\phi)$ and $Q(\phi)$ map $\breve{H}$ into itself but also that they can be defined for any smooth function $\phi$ on $S^{1}$. That proves

Proposition (4.3) The Lie algebra of smooth maps $S^{1} \rightarrow s u_{2}$ acts projectively on $\breve{H}$.

We can now complete the proof of (4.1). We have seen that the operators $A(\phi)$ can be exponentiated to give unitary transformations of $H$. The same must therefore hold for $P(\phi)$ and $Q(\phi)$. This gives us projective unitary representations of three subgroups of $\operatorname{Map}\left(S^{1} ; S U_{2}\right)$. The three subgroups clearly generate the whole group. To see that they fit together to give a projective unitary representation of $\operatorname{Map}\left(S^{1} ; S U_{2}\right)$ one need only observe that if $f \in \operatorname{Map}\left(S^{1} ; S U_{2}\right)$ is expressed in two different ways in terms of elements of the three subgroups, leading to two operators $U_{f}$ and $U_{f}^{\prime}$ representing it, then $U_{f}^{-1} U_{f}^{\prime}$ commutes with the action of the Lie algebra $\operatorname{Map}\left(S^{1} ; s u_{2}\right)$. As this action is irreducible $U_{f}$ and $U_{f}^{\prime}$ can differ only by a scalar.

\section{Generalization to other groups}

Suppose that $G$ is a compact Lie group with a maximal torus $T$. The discussion in this section can be generalized to prove that in certain circumstances a projective representation of $\operatorname{Map}\left(S^{1} ; T\right)$ can be extended canonically to one of $\operatorname{Map}\left(S^{1} ; G\right)$.

The method seems to apply only to groups $G$ which are simply laced. Let us recall that $G$ is simply laced if there is an invariant inner product on its Lie algebra $\mathfrak{g}$ in terms of which all the roots have the same length. (Equivalently one can say: if the Weyl group of $G$ acts transitively on the roots.) This happens if and only if $G$ is a product of circles and simple groups of types $A, D$ and $E$, i.e. if it has no factors of type, $B, C, F$ or $G$. I shall also assume that $G$ is simply connected, although that is not essential.

Proposition (4.4) Suppose that $G$ is simply laced and simply connected with maximal torus $T$. Let $H$ be a projective unitary representation of $M=\operatorname{Map}\left(S^{1} ; T\right)$ with the cocycle $c$ described below. Suppose that $H$ is symmetric with positive energy, and that each eigenspace $H(q)$ of the rotations of the circle is finite dimensional. Then the action of $M$ on $H$ extends canonically to an action of $\operatorname{Map}\left(S^{1} ; G\right)$.

To define the cocycle $c$ I need to recall some facts about simply laced groups.

We write $T=t / 2 \pi L$, where $t$ is the Lie algebra of $T$, and $L$ is a lattice in $t$. There is an exact sequence

$$
0 \rightarrow L \stackrel{\times 2 \pi}{\rightarrow} \operatorname{Map}\left(S^{1} ; t\right) \rightarrow \operatorname{Map}\left(S^{1} ; T\right) \rightarrow L \rightarrow 0 .
$$

Let $F$ denote the smooth function $f: \mathbb{R} \rightarrow t$ such that

$$
\Delta_{f}=\frac{1}{2 \pi}(f(\theta+2 \pi)-f(\theta))
$$

is constant; and let $F_{L}=\left\{f \in F: \Delta_{f} \in L\right\}$. Then $\operatorname{Map}\left(S^{1} ; \mathrm{T}\right)=F_{L} / 2 \pi L$.

The roots of $G$ are certain linear maps $\alpha: t \rightarrow \mathbb{R}$. For each $\alpha$ we define $h_{\alpha} \in t$ so that $\alpha(\xi)=\left\langle h_{\alpha}, \xi\right\rangle$ for all $\xi \in t$. For a simply laced group the inner product $\langle$, $\rangle$ on $g$ can be normalized so that $\left\langle h_{\alpha}, h_{\alpha}\right\rangle=2$ for each root $\alpha$. (In the case of $S U_{n}$ this amounts to defining $\langle\xi, \eta\rangle=-\operatorname{trace}(\xi \eta)$, and in the case of $S O_{2 n}$ it corresponds 
to $\langle\xi, \eta\rangle=-\frac{1}{2}$ trace $(\xi \eta)$.) It is well known that each $h_{\alpha}$ belongs to the lattice $L$, and for a simply connected group the $h_{\alpha}$ even generate $L$ and are precisely the set of all $\lambda \in L$ such that $\langle\lambda, \lambda\rangle=2$. In that case, $\lambda \mapsto \frac{1}{2}\langle\lambda, \lambda\rangle$ is an integer-valued quadratic form on $L$. Let us choose a bilinear form

$$
\sigma: L \times L \rightarrow \mathbb{Z} / 2 \text { such that } \sigma(\lambda, \lambda) \equiv \frac{1}{2}\langle\lambda, \lambda\rangle \quad(\bmod 2) .
$$

Then

$$
\sigma(\lambda, \mu)+\sigma(\mu, \lambda) \equiv\langle\lambda, \lambda\rangle(\bmod 2)
$$

for all $\lambda, \mu \in L$.

The complexified Lie algebra $\mathfrak{g}_{\mathbb{C}}$ can be decomposed

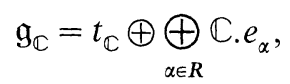

where $R$ is the set of roots. The relations are

$$
\begin{aligned}
& {\left[\xi, e_{\alpha}\right]=\alpha(\xi) e_{\alpha} \text { for } \xi \in t_{\mathbb{C}}} \\
& {\left[e_{\alpha}, e_{-\alpha}\right]=h_{\alpha}} \\
& {\left[e_{\alpha}, e_{\beta}\right]=0 \text { if } \alpha \neq-\beta \text { and }\langle\alpha, \beta\rangle \neq-1,} \\
& {\left[e_{\alpha}, e_{\beta}\right]=(-1)^{\sigma(\alpha, \beta)} e_{\alpha+\beta} \text { if }\langle\alpha, \beta\rangle=1 .}
\end{aligned}
$$

(Here $\sigma(\alpha, \beta)$ means $\sigma\left(h_{\alpha}, h_{\beta}\right)$. These are not quite the usual form of the generators and relations for $\mathfrak{g}$, but are easily checked to be equivalent to them. Notice that the choice of $\sigma$ is immaterial, for if $\sigma^{\prime}$ is another choice then $\sigma^{\prime}(\lambda, \mu)-\sigma(\lambda, \mu)=$ $\zeta(\lambda+\mu)-\zeta(\lambda)-\zeta(\mu)$ for some $\zeta: L \rightarrow \mathbb{Z} / 2$.)

We define a blinear from $s: F \times F \rightarrow \mathbb{R}$ by

$$
s(f, g)=\frac{1}{4 \pi} \int_{0}^{2 \pi}\left\langle f^{\prime}(\theta), g(\theta)\right\rangle d \theta+\frac{1}{2}\left\langle\Delta_{f}, g(0)\right\rangle .
$$

If $g$ is constant then $s(f, g)=\left\langle\Delta_{f}, g\right\rangle$, and if $f$ is constant then $s(f, g)=0$, so $s\left(\left(2 \pi L \times F_{L}\right)+\left(F_{L} \times 2 \pi L\right)\right) \subset 2 \pi \mathbb{Z}$, and we can define a cocycle $c$ on $M=$ $\operatorname{Map}\left(S^{1} ; T\right)$ by

$$
c\left(e^{i f}, e^{i g}\right)=(-1)^{\sigma\left(\Delta_{f}, \Delta_{g}\right)} e^{-i s(f, g)}
$$

Suppose now that $e^{i f} \mapsto T\left(e^{i f}\right)$ is a projective unitary representation of $M$ on a Hilbert space $H$, associated to the cocycle $c$. Passing to the Lie algebra of $M$ gives us unbounded operators $A(f)$ on $H$ for each $f: S^{1} \rightarrow t$, and we have

$$
[A(f), A(g)]=2 i s(f, g) \text {. }
$$

As before, we assume that the group of rotations of the circle acts on $H$ with positive energy, compatibly with the action of $M$.

Let us identify the roots of $G$ with the vectors $\xi$ of length 2 in the lattice $L$. To each $\xi$, and $\theta \in S^{1}$, we associate a "blip" $\gamma_{\theta, \lambda}^{\xi}=\exp \left(\xi \cdot f_{\theta, \lambda}\right) \in M$, where $0 \leqq \lambda<1$ and $f_{\theta, \lambda}: \mathbb{R} \rightarrow \mathbb{R}$ is as on page 8 . We find just as before that $\left(-\lambda^{2}\right)^{-1} T\left(\gamma_{\theta, \lambda}^{\xi}\right)$ tends to an operator $B_{\theta}^{\xi}: \breve{H} \rightarrow \hat{H}$ as $\lambda \rightarrow 1$, where

$$
B_{\theta}^{\xi}=T\left(e^{i \xi \xi_{\theta}}\right) e^{i A\left(\xi f_{\theta}^{+}\right)} e^{i A\left(\xi_{\theta}^{-}\right)} .
$$


For a trigonometrical polynomial $\phi: S^{1} \rightarrow \mathbb{C}$ we define

$$
B^{\xi}(\phi)=\frac{1}{2 \pi} \int_{0}^{2 \pi} \phi(\theta) B_{\theta}^{\xi} d \theta: \check{H} \rightarrow \check{H} .
$$

Proposition (4.2) generalizes to

Proposition (4.5) (i) $\left[A(f), B^{\xi}(\phi)\right]=B^{\xi}(\langle f, S \phi\rangle)$,

(ii) $\left[B^{\xi}(\phi), B^{-\xi}(\psi)\right]=A(\xi \phi \psi)+i S(\phi, \psi)$,

(iii) $\left[B^{\xi}(\phi), B^{\eta}(\psi)\right]=0$ if $\xi \neq-\eta$ and $\langle\xi, \eta\rangle \neq-1$,

(iv) $\left[B^{\xi}(\phi), B^{\eta}(\psi)\right]=(-1)^{\sigma(\xi, \eta)} B^{\xi+\eta}(\phi \psi)$ if $\langle\xi, \eta\rangle=-1$.

These are the commutation relations for a projective representation of the Lie algebra $\operatorname{Map}_{\mathrm{alg}}\left(S^{1} ; \mathfrak{g}_{\mathbb{C}}\right)$ corresponding to the cocycle

$$
(f, g) \mapsto \omega(f, g)=\frac{1}{2 \pi} \int_{0}^{2 \pi}\left\langle f^{\prime}(\theta), g(\theta)\right\rangle d \theta .
$$

The only comment worth making about the proof of (4.5) is that in proving (iv) one proceeds exactly as in the proof of (4.2) (iii), and obtains a formula for $\left[B_{\theta}^{\lambda, \xi}, B_{\phi}^{\lambda, \eta}\right]$ where the expression in braces in the earlier proof in replaced by

$$
\begin{gathered}
(-1)^{\sigma(\xi, \eta)}\left\{\frac{e^{(1 / 2) i(\theta-\phi)}}{1-\lambda^{2} e^{i(\theta-\phi)}}+\frac{e^{-(1 / 2) i(\theta-\phi)}}{1-\lambda^{2} e^{-i(\theta-\phi)}}\right\} \\
=(-)^{\sigma(\xi, \eta)} \frac{2\left(1-\lambda^{2}\right) \cos \frac{1}{2}(\theta-\phi)}{1-2 \lambda^{2} \cos (\theta-\phi)+\lambda^{4}} .
\end{gathered}
$$

This tends to $(-1)^{\sigma(\xi, \eta)} 2 \pi \delta(\theta-\phi)$ as $\lambda \rightarrow 1$, which gives the formula we want.

Extending the representation of the Lie algebra $\operatorname{Map}_{\text {alg }}\left(S^{1} ; \mathfrak{g}_{\mathbb{C}}\right)$ to one of the group $\operatorname{Map}\left(S^{1} ; G\right)$ is done just as when $G=S U_{2}$, and presents nothing new.

It is worth considering how many representations of $\Gamma=\operatorname{Map}\left(S^{1} ; G\right)$ - or, more precisely, of its central extension $\hat{\Gamma}$-we obtain by this method. They correspond to representations of $\hat{M}$ which are symmetric and of positive energy. These are constructed and classified exactly as in Sect. $3 . \hat{M}$ is a semidirect product $L \times \hat{M}_{0}$, where $\hat{M}_{0}$ is the identity component. As before $M_{0} \cong T \times V$, where $V=\operatorname{Map}\left(S^{1} ; t\right) /($ constants) has a nondegenerate skew form induced by $s$. The arguments of Sect. 3. show that $\hat{M}_{0}$ has a unique irreducible representation $H_{\chi}$ of the appropriate kind for each character $\chi$ of $T$, in which $T$, which is in the centre of $\widehat{M}_{0}$, acts scalarly by $\chi$. The character group of $T$ is $L^{*}=\operatorname{Hom}(L ; \mathbb{Z})$. Conjugation by $\lambda \in L$ transforms $H_{\chi}$ to $H_{\chi+\lambda}$, where $L$ is embedded in $L^{*}$ by the inner product $\langle$,$\rangle . Thus we obtain an irreducible representation \bigoplus_{\lambda \in L} H_{\chi+\lambda}$ of $\hat{M}$ for each coset

$\chi+L$ of $L$ in $L^{*}$. Now $L^{*} / L$ is precisely the centre $Z$ of $G$. The common centre of $\hat{M}$ and $\hat{\Gamma}$ is $Z \times \mathbb{T}$, and the representations we have obtained are classified by the action of $Z$. (By using the inner product we have identified $Z=L^{*} / L$ with its character group.)

These conclusions agree with Kač's theory of the representation of $\operatorname{Map}\left(S^{1} ; G\right)$, because $L^{*} / L$ is precisely the set of orbits of the lattice of weights $L^{*}$ under the affine Weyl group of $G$. 


\section{The Metaplectic Representation}

We have seen that the group $\operatorname{Diff}\left(S^{1}\right)$ is a group of automorphisms of $\hat{M}$. Our next task is to show that it acts projectively by unitary transformations on $H$, intertwining with the action of $\hat{M}$. As the representation of $\widehat{M}$ on $H$ is irreducible this would follow from Schur's Lemma if one knew that the isomorphism class of the representation of $\hat{M}$ on $H$ did not change when twisted by a diffeomorphism: but unfortunately that is not clear, as it is not obvious that the property of having positive energy is preserved.

The action of $\operatorname{Diff}\left(S^{1}\right)$ on $\hat{M}$ leaves fixed the constants $C \subset M$, so $\operatorname{Diff}\left(S^{1}\right)$ ought to act independently in each eigenspace $H_{2 k}$ of $C$. We shall show that it acts on $H_{0}=\hat{S}(W)$. It is then automatic that it acts on all of $H$ because $H$ is the representation of $\hat{M}$ induced from the representation $H_{0}$ of $\hat{M}_{0}$, and $\hat{M} / \hat{M}_{0} \cong$ $\left(\operatorname{Diff}\left(S^{1}\right) \tilde{\times} \hat{M}\right) /\left(\operatorname{Diff}\left(S^{1}\right) \tilde{\times} \hat{M}_{0}.\right)$

$\operatorname{Diff}\left(S^{1}\right)$ acts on $V=\operatorname{Map}\left(S^{1} ; \mathbb{R}\right) / \mathbb{R}$ preserving the skew form $S$, so it can be thought of as a subgroup of the symplectic group $S p(V)$. If $V$ were finite-dimensional then a double converging of $S p(V)$ would act, by the metaplectic representation, on $\hat{S}(W)$. In the infinite-dimensional case one must replace $S p(V)$ by a subgroup $S p_{0}(V)$ consisting of maps which are not too far from preserving the polarization $V_{\mathbb{C}}=W \oplus \bar{W}$. The definition, which is due to Shale [10], is as follows.

Let us suppose that we have a real vector space $V$ with a skew form $S: V \times V \rightarrow \mathbb{R}$ and a positive polarization $V_{\mathbb{C}}=W \oplus \bar{W}$. As usual we regard $W$ as a pre-Hilbertspace with $\left\langle w_{1}, w_{2}\right\rangle=2 i S\left(\bar{w}_{1}, w_{2}\right)$. With respect to the decomposition $V_{\mathbb{C}}=W \oplus \bar{W}$ an endomorphism $A$ of $V$ can be expressed as a matrix

$$
A=\left(\begin{array}{ll}
a & b \\
\bar{b} & \bar{a}
\end{array}\right) .
$$

We find that $A$ belongs to $S p(V)$ if and only if

(i) $\bar{a}^{t} a-b^{t} \bar{b}=1$, and

(ii) $\bar{a}^{t} b$ is symmetric.

(These equations should be thought of as shorthand: they do not really presuppose the existence of the transposed operators.)

Definition (5.1) $S p_{0}(V)$ is the subset of $S p(V)$ consisting of elements $A$ as above such that $b: \bar{W} \rightarrow W$ is a Hilbert-Schmidt operator.

("Hilbert-Schmidt" means that $\sum\left\|b \bar{\varepsilon}_{k}\right\|^{2}$ converges for any orthonormal family $\left\{\bar{\varepsilon}_{k}\right\}$ in $\overline{\mathrm{W}}$.)

If $A \in S p_{0}(V)$ it follows from (i) above that $a: W \rightarrow W$ is a bounded operator, and hence that $S p_{0}(V)$ is a subgroup of $S p(V)$, for the Hilbert-Schmidt operators are closed under composition with bounded operators.

Proposition (5.2) (Shale) $S p_{0}(V)$ acts projectively by unitary transformations on $\hat{S}(W)$.

Before proving this I shall show that $S p_{0}(V)$ does contain $\operatorname{Diff}\left(S^{1}\right)$, using a simple argument pointed out to me by Kazhdan.

Proposition (5.3) $\operatorname{Diff}\left(S^{1}\right) \subset S p_{0}(V)$.

Proof. Let us write $\left\{\varepsilon_{n}\right\}$ for the standard basis $\left\{e^{i n \theta}\right\}_{n \neq 0}$ of $V_{\mathbb{C}}=\operatorname{Map}\left(S^{1} ; \mathbb{C}\right) / \mathbb{C}$. 
If $\phi \in \operatorname{Diff}\left(S^{1}\right)$ we write $\left\{\lambda_{n m}\right\}$ for its matrix elements with respect to this basis, i.e. $\phi^{*}\left(\varepsilon_{n}\right)=\sum \lambda_{n m} \varepsilon_{m}$. We have to show that the matrix $\left\{\lambda_{n,-m}\right\}_{n, m>0}$ defines a Hilbert -Schmidt operator. Clearly it is enough to show that $\left\{\lambda_{n,-m}\right\}$ is rapidly decreasing, in the sense that for each integer $k$ we have $\left|\lambda_{n,-m}\right| \leqq C(n+m)^{-k}$ for some constant $C$ (depending on $k$ ).

Now

$$
\lambda_{n,-m}=\frac{1}{2 \pi} \int_{0}^{2 \pi} e^{i n \phi(\theta)+i m \theta} d \theta
$$

(Here $\phi$ is regarded as a map $\phi: \mathbb{R} \rightarrow \mathbb{R}$ such that $\phi(\theta+2 \pi)=\phi(\theta)+2 \pi$.) For any $t \in[0,1]$ the function $\phi_{t}$ defined by $\phi_{t}(\theta)=t \phi(\theta)+(1-t) \theta$ is also a diffeomorphism, and when $t=n /(n+m)$ we have

$$
\begin{aligned}
\lambda_{n,-m} & =\frac{1}{2 \pi} \int_{0}^{2 \pi} e^{i(n+m) \phi_{t}(\theta)} d \theta \\
& =\frac{1}{2 \pi} \int_{0}^{2 \pi} e^{i(n+m) \theta} \psi_{t}^{\prime}(\theta) d \theta
\end{aligned}
$$

where $\psi_{t}$ is the inverse function to $\phi_{t}$. So

$$
\lambda_{n,-m}=\frac{i^{k}}{2 \pi} \cdot(n+m)^{-k} \int_{0}^{2 \pi} e^{i(n+m) \theta} \psi_{t}^{(k+1)}(\theta) d \theta
$$

on integrating by parts $k$ times, and

$$
\left|\lambda_{n,-m}\right| \leqq(n+m)^{-k} \sup \left\{\left|\psi_{t}^{(k+1)}(\theta)\right|: 0 \leqq \theta \leqq 2 \pi, 0 \leqq t \leqq 1\right\},
$$

as we want.

To construct the metaplectic representation of $S p_{0}(V)$ I shall introduce an infinite-dimensional analogue of the Siegel bounded complex domain $S p_{2 n}(R) / U_{n}$. Let us recall that $S p_{2 n}(R) / U_{n}$ can be realized as the space of complex symmetric $n \times n$ matrices $Z$ such that $\bar{Z} Z<1$.

Let $X$ denote the space of symmetric Hilbert-Schmidt operators $Z: \bar{W} \rightarrow W$ such that $\bar{Z} Z<1$, i.e. those such that

(i) $S\left(\bar{w}_{1}, Z \bar{w}_{2}\right)=S\left(\bar{w}_{2}, Z \bar{w}_{1}\right)$, and

(ii) $1-\bar{Z} Z$ is positive-definite.

The group $S p_{0}(V)$ acts transitively on $X$ by the formula

$$
\left(\begin{array}{ll}
a & b \\
\bar{b} & \bar{a}
\end{array}\right) \cdot Z=(a Z+b)(\bar{b} Z+\bar{a})^{-1}:
$$

the proof is the same as in the finite-dimensional case. The stabiliser of $0 \in X$ is the unitary group $U(W)$, so $X \cong S p_{0}(V) / U(W)$.

In the finite-dimensional case $S p_{2 n}(R) / U_{n}$ can be identified with the set of all positive polarizations of $V$. In our case $X$ is to the thought of as the set of positive polarizations of $V$ which are not too far away from $W$. (One gets a polarization $V_{\mathbb{C}}=U \oplus \bar{U}$ from $Z: \bar{W} \rightarrow W$ by taking $\bar{U}$ to be the graph of $Z$.)

The symmetric Hilbert-Schmidt operators $Z: \bar{W} \rightarrow W$ can be regarded as 
elements of $\hat{S}^{2}(W)$ by identifying $Z$ with $\sum \varepsilon_{k} \cdot Z \bar{\varepsilon}_{k}$, where $\left\{\varepsilon_{k}\right\}$ is an orthonormal basis for $W$. For each $Z \in X$ we consider the element $e^{(1 / 2) z}$ of $\hat{S}(W)$

Lemma (5.4) $\left\langle e^{(1 / 2) Z_{1}}, e^{(1 / 2) Z_{2}}\right\rangle=\operatorname{det}\left(1-\bar{Z}_{1} Z_{2}\right)^{-1 / 2}$

(Here one chooses the branch of the square root which is +1 when $\hat{Z}_{1} Z_{2}=0$. Notice also that the determinant is defined, because $\bar{Z}_{1} Z_{2}$, being the product of two Hilbert-Schmidt operators, is of trace class.)

It is enough, by continuity, to prove (5.4) when $Z_{1}$ and $Z_{2}$ have finite rank, in which case I shall leave it as an exercise for the reader.

In view of (5.4) the map $Z \mapsto \varepsilon_{Z}=\operatorname{det}(1-\bar{Z} Z)^{1 / 4} e^{(1 / 2) Z}$ is an embedding of the domain $X$ into the space of unit vectors in $S(W)$, and

$$
\left\langle\varepsilon_{Z_{1}}, \varepsilon_{Z_{2}}\right\rangle=\operatorname{det}\left(1-\bar{Z}_{1} Z_{1}\right)^{1 / 4} \operatorname{det}\left(1-\bar{Z}_{2} Z_{2}\right)^{1 / 4} \operatorname{det}\left(1-\bar{Z}_{1} Z_{2}\right)^{-1 / 2} \ldots
$$

Now let $F_{X}$ denote the free vector space generated by the symbols $\left\{\varepsilon_{Z}\right\}_{Z \in X}$, and let $H_{X}$ be the Hilbert space obtained by completing $F_{X}$ using the inner product defined by the formula $(*)$. (This inner product on $F_{X}$ is positive because it is induced from that of $\hat{S}(W)$.) Clearly $H_{X}$ is a closed subspace of $\hat{S}(W)$.

Proposition (5.5) $H_{X}=\hat{S}^{\mathrm{even}}(W)$

I shall postpone the proof of this for the moment.

For each $A \in S p_{0}(V)$ one can define a unitary operator $T_{A}: H_{X} \rightarrow H_{X}$ by

$$
T_{A} \cdot \varepsilon_{Z}=\mu \operatorname{det}\left(1+\bar{a}^{-1} \bar{b} Z\right)^{1 / 2} \cdot \varepsilon_{A Z^{\prime}}
$$

where $\mu: \mathbb{C}^{\times} \rightarrow \mathbb{T}$ is radial projection. To see that $T_{A}$ is well-defined one must check that

$$
\left\langle\varepsilon_{Z_{1}}, \varepsilon_{Z_{2}}\right\rangle=\mu \operatorname{det}\left(1+\bar{a}^{-1} \bar{b} Z_{1}\right)^{-1 / 2} \mu \operatorname{det}\left(1+\bar{a}^{-1} \bar{b} Z_{2}\right)^{1 / 2}\left\langle\varepsilon_{A Z_{1}}, \varepsilon_{A Z_{2}}\right\rangle,
$$

which, however, is a simple calculation. It is also straightforward to check that

$$
T_{A_{1}} T_{A_{2}}=c\left(A_{1}, A_{2}\right) T_{A_{1} A_{2}},
$$

where $c\left(A_{1}, A_{2}\right)=\operatorname{det}\left(a_{1}^{-1} a_{3} a_{2}^{-1}\right)^{-1 / 2}$. Here

$$
A_{i}=\left(\begin{array}{cc}
a_{i} & b_{i} \\
\bar{b}_{i} & \bar{a}_{i}
\end{array}\right)
$$

for $i=1,2,3$, and $A_{3}=A_{1} A_{2}$. The determinant is well-defined because $a_{1}^{-1} a_{3} a_{2}^{-1}=1+a_{1}^{-1} b_{1} \bar{b}_{2} a_{2}^{-1}$ is of the form $1+$ (trace class), and $\left\|a_{1}^{-1} b_{1} \bar{b}_{2} a_{2}^{-1}\right\|<1$.

The cocycle $c$ evidently measures the extent by which $A \mapsto a$ fails to be a homomorphism, i.e. by which $A$ fails to preserve the polarization $V_{\mathbb{C}}=W \oplus \bar{W}$. I shall return to this point in Sect. 7.

To understand what is going on in the preceding formulae one should adopt the following point of view. There is a natural holomorphic line bundle $L$ on $X=$ \{polarizations of $V$ \} whose fibre at $U\left(\right.$ where $\left.V_{\mathbb{C}}=U \oplus \bar{U}\right)$ is $\operatorname{det}(U)^{-1 / 2}$. We have chosen a trivialization of $L$ by identifying $U$ with $W$ by $U \subset V_{\mathbb{C}} \stackrel{p r}{\longrightarrow} W$. In the finite dimensional case the action of $S p_{2 n}(\mathbb{R})$ on $X$ is covered by an action of a double covering $\widetilde{S} p_{2 n}(\mathbb{R})$ on $L$, which in terms of our trivialization is given by

$$
A \cdot(Z, \lambda)=\left(A \cdot Z, \mu \operatorname{det}(\bar{b} Z+\bar{a})^{1 / 2} \cdot \lambda\right) .
$$


An element of $\tilde{S} p_{2 n}(\mathbb{R})$ is an element of $S p_{2 n}(\mathbb{R})$ together with a choice of a square root of det $(a)$. But in our case we cannot define det $(a)$, let alone its square root; so we are forced to make an extension $\widetilde{S} p_{0}(V)$ of $S p_{0}(V)$ by $\mathbb{T}$ act on $L$ by

$$
A \cdot(Z, \lambda)=\left(A \cdot Z, \mu \operatorname{det}\left(1+\bar{a}^{-1} \bar{b} Z\right)^{1 / 2} \cdot \lambda\right)
$$

Instead of embedding $X$ in $\hat{S}(W)$ above it would have been more natural to embed the line bundle $L$ linearly in $\hat{S}(W)$. The action of $\widetilde{S} p_{0}(V)$ is then extended by linearity to $\hat{S}^{\text {even }}(W)$.

We have now made $S p_{0}(V)$ act projectively on $\hat{S}^{\text {even }}(W)$. We can extended the action to $\hat{S}^{\text {odd }}(W)$ by the following device. Consider $S p_{0}\left(V \oplus \mathbb{R}^{2}\right)$, where $\mathbb{R}^{2}$ has the obvious skew form. This contains $S p_{0}(V) \times \mathbb{T}$, where $\mathbb{T}=S O_{2} \subset S p\left(\mathbb{R}^{2}\right)$. There is a polarization $\left(V \oplus \mathbb{R}^{2}\right)_{\mathbb{C}}=(W \oplus \mathbb{C}) \oplus(\bar{W} \oplus \overline{\mathbb{C}})$ where $\mathbb{T}$ acts in the natural way on $\mathbb{C}$. Then $S p_{0}(V) \times \mathbb{T}$ acts on

$$
\hat{S}^{\text {even }}(W \oplus \mathbb{C})=\hat{S}^{\text {even }}(W) \hat{\otimes} \hat{S}^{\text {even }}(\mathbb{C}) \oplus \hat{S}^{\text {odd }}(W) \hat{\otimes} \hat{S}^{\text {odd }}(\mathbb{C}),
$$

and $S p_{0}(V)$ acts in each isotypical component of the action of $\mathbb{T}$. The action on $\widehat{S}^{\text {odd }}(W) \otimes S^{k}(\mathbb{C}) \cong \widehat{S}^{\text {odd }}(W)$, for any odd $k$, gives us what we want.

It remains to give the proof of (5.5).

Clearly $H_{X}$ contains $e^{t w w}$ for each $w \in W$ and $t \in \mathbb{R}$. By differentiating this repeatedly with respect to $t$ at $t=0$ we find, because $H_{X}$ is a closed subspace, that it contains $w^{2 k}$ for each $k$. Then from the identity

$$
m ! w_{1} w_{2} \ldots w_{m}=\sum_{\sigma}(-1)^{m-|\sigma|}\left(\sum_{i \in \sigma} w_{i}\right)^{m},
$$

where $\sigma$ runs through the subsets of $\{1,2, \ldots, m\}$, and $|\sigma|$ denotes the number of elements in $\sigma$, we find that $H_{X}$ contains $S^{m}(W)$ whenever $m$ is even.

A possible disadvantage of the method adopted here for constructing the metaplectic representation is that it does not make manifest that the action of $S p_{0}(V)$ intertwines correctly with the unbounded operators $A(v)$ for $v \in V$, and hence with the group $M$ of Sect. 2. The rest of this section is devoted to establishing that it does so.

We begin by considering the action of the Lie algebra $s p_{0}(V)$ of $S p_{0}(V)$ on $\widehat{S}(W)$. An element of $s p_{0}(V)$ is a matrix

$$
\left(\begin{array}{cc}
\alpha & \beta \\
\bar{\beta} & \bar{\alpha}
\end{array}\right)
$$

where $\alpha$ is bounded and skew-Hermitian and $\beta$ is symmetric and Hilbert-Schmidt. The complexification $s p_{0}^{\mathbb{C}}(V)$ consists of operators

$$
\left(\begin{array}{cc}
\alpha & \beta \\
\bar{\gamma} & -\alpha^{t}
\end{array}\right)
$$

where $\alpha$ is an arbitrary bounded operator and $\beta$ and $\gamma$ are symmetric HilbertSchmidt operators.

Proposition (5.6) The element

$$
\left(\begin{array}{cc}
\alpha & \beta \\
\bar{\gamma} & -\alpha^{t}
\end{array}\right)
$$


of $s p_{0}^{\mathbb{C}}(V)$ acts on $\hat{S}(W)$ as $D_{\alpha}+\frac{1}{2} M_{\beta}+\frac{1}{2} M_{\gamma}^{*}$, where $D_{\alpha}$ is the derivation of $\hat{S}(W)$ induced by $\alpha: W \rightarrow W, M_{\beta}$ is multiplication by $\beta \in \hat{S}^{2}(W)$, and $M_{\gamma}^{*}$ is the adjoint of $M_{\gamma}$. Proof. One can consider separately elements of the forms

$$
\left(\begin{array}{cc}
\alpha & 0 \\
0 & -\alpha^{t}
\end{array}\right),\left(\begin{array}{ll}
0 & \beta \\
0 & 0
\end{array}\right) \text {, and }\left(\begin{array}{ll}
0 & 0 \\
\bar{\gamma} & 0
\end{array}\right)
$$

For the first kind, which preserve the polarization $V_{\mathbb{C}}=W \oplus \bar{W}$, the result is clear.

By direct calculation one finds that the effect of

$$
\left(\begin{array}{cc}
0 & \beta \\
\bar{\beta} & 0
\end{array}\right) \in \operatorname{sp}_{0}(V)
$$

on $e^{(1 / 2) Z} \in \hat{S}(W)$ is multiplication by $\frac{1}{2}(\beta-Z \bar{\beta} Z-\operatorname{tr}(\bar{\beta} Z))$. It follows that the action of

$$
\left(\begin{array}{ll}
0 & \beta \\
0 & 0
\end{array}\right)
$$

on $e^{(1 / 2) Z}$, and hence on all of $\widehat{S}(W)$, is multiplication by $\frac{1}{2} \beta$. Finally, in any unitary representation

$$
\left(\begin{array}{ll}
0 & 0 \\
\bar{\gamma} & 0
\end{array}\right)
$$

has to be represented by the adjoint of the representative of

$$
\left(\begin{array}{ll}
0 & \gamma \\
0 & 0
\end{array}\right)
$$

Returning now to the intertwining of $s p_{0}(V)$ with the operators $A(v)$ for $v \in V$, what we want to show is that

$$
[X, A(v)]=A(X v)
$$

for $X \in s p_{0}^{\mathbb{C}}(V)$ and $v \in V_{\mathbb{C}}$. Because $A(\bar{v})=A(v)^{*}$ it is enough to consider [X, $\left.A(w)\right]$ for $w \in W$. When $X$ is of the form

$$
\left(\begin{array}{cc}
\alpha & 0 \\
0 & -\alpha^{t}
\end{array}\right)
$$

the result is obvious. When

$$
X=\left(\begin{array}{cc}
0 & \beta \\
0 & 0
\end{array}\right)
$$

then $X w=0$; but on the left we have two multiplication operators, so the commutator vanishes too. When

$$
X=\left(\begin{array}{ll}
0 & 0 \\
\bar{\gamma} & 0
\end{array}\right)
$$

then $X w=\bar{\gamma} w \in \bar{W}$, and we have to show that $[X, A(w)]=A(\gamma \bar{w})^{*}$. By adjunction this is equivalent to $\left[A(w)^{*}, X^{*}\right]=A(\gamma \bar{w})$. Now $X^{*}$ is multiplication by $\frac{1}{2} \gamma$ and 
$A(w)^{*}$ is a derivation. So the commutator is multiplication by $\frac{1}{2} A(w)^{*} \gamma$, which is precisely $\bar{w}$. That completes the proof.

Finally we come to the group $S p_{0}(V)$. It is of course not true that the elements of this group can be obtained by exponentiating bounded operators in the Lie algebra $s p_{0}(V)$. On the other hand $S p_{0}(V)$ has a normal subgroup $S p_{c p t}(V)$ consisting of elements which differ from the identity by a compact operator. The exponentials of bounded operators clearly generate $S p_{c p t}(V)$, so for this group the desired intertwining with the $A(v)$ follows from what we have proved. $S p_{0}(V)$ also contains the unitary group $U(W)$ of $W$, the transformations which preserve the polarization $V_{\mathbb{C}}=W \oplus \bar{W}$. For these the intertwining relation is obvious. But that is all we need, in view of

Proposition (5.7) $S p_{0}(V)=S p_{c p t}(V) \cdot U(W)$.

Proof. We have already remarked that $S p_{0}(V) / U(W)$ is the space of symmetric Hilbert-Schmidt operators $\bar{W} \rightarrow W$, so it is enough to see that $S p_{c p t}(V)$ acts transitively on $X$. But that is obvious.

\section{The Action of Diff $\left(S^{1}\right)$ on $H$}

In the last section we showed that $\operatorname{Diff}\left(S^{1}\right)$ acts projectively on $H$, intertwining with the action of $\operatorname{Map}\left(S^{1} ; S U_{2}\right)$. It follows that the product $\operatorname{Diff}\left(S^{1}\right) \times S U_{2}$ acts on $H$. In this section I shall discuss the decomposition of $H$ under this action.

I shall write $A, B, B^{*}$ for the operators $A(1), B(1), B(1)^{*}$ of Sect. 4 . They form a basis for the complexification of the Lie algebra of $S U_{2}$, and satisfy

$$
\begin{aligned}
& {[A, B]^{*}=2 B,} \\
& {\left[A, B^{*}\right]=-2 B^{*},} \\
& {\left[B, B^{*}\right]=A .}
\end{aligned}
$$

Recall that $H=\bigoplus H_{2 k}$, where $H_{2 k}$ is the $2 k$-eigenspace of $A$. Thus $B\left(H_{2 k}\right) \subset H_{2 k+2}$ and $B^{*}\left(H_{2 k}\right) \subset H_{2 k-2}$.

From the elementary representation theory of $\mathrm{SU}_{2}$ we know that we can write

$$
H=\bigoplus_{q=0,1,2, \ldots} P_{q^{2}} \otimes D_{q},
$$

where $D_{q}$ is the irreducible representation of $S U_{2}$ of dimension $2 q+1$, and $P_{q^{2}}=\left\{\xi \in H_{2 q}: B \xi=0\right\}$ is a representation of $\operatorname{Diff}\left(S^{1}\right)$. Furthermore the operator $B^{*}: H_{2 k+2} \rightarrow H_{2 k}$ is injective when $k \geqq 0$, and

$$
H_{2 k}=P_{k^{2}} \oplus B^{*}\left(H_{2 k+2}\right) \text {. }
$$

It follows that $H_{2 k} \cong \underset{q \geqq k}{\bigoplus} P_{q^{2}}$ as a representation of $\operatorname{Diff}\left(S^{1}\right)$.

Each space $H_{2 k}$ is graded by the action of the group $R$ of rotations of $S^{1}: H_{2 k}=\bigoplus_{q \geqq 0} H_{2 k}(q)$. Let $\pi_{2 k}(t)=\sum \operatorname{dim} H_{2 k}(q) \cdot t^{q}$ be the Poincaré series of $H_{2 k}$. We know that $\pi_{2 k}(t)=t^{k^{2}} \pi_{0}(t)$, and that $\pi_{0}=\pi$ is the partition function:

$$
\pi(t)=\prod_{n>0}\left(1-t^{n}\right)^{-1}
$$


From $\left(^{*}\right)$ we find

Proposition (6.1) The Poincaré series of $P_{q^{2}}$ is $t^{q^{2}}\left(1-t^{2 q+1}\right) \pi(t)$.

Kač has proved the conjecture of Goldstone that the representations $P_{q^{2}}$ of $\operatorname{Diff}\left(S^{1}\right)$ are irreducible. Here I shall prove the irreducibility only for $P_{0}$. (But cf. Prop. (6.7).) Before doing so it seems appropriate to make some elementary general remarks about the representation theory of the Lie algebra of $\operatorname{Diff}\left(S^{1}\right)$, i.e. the Lie algebra $\mathscr{V}=\operatorname{Vect}\left(S^{1}\right)$ of smooth vector fields on the circle.

The complexification $\mathscr{V}_{\mathbb{C}}$ has an obvious basis $\left\{v_{k}\right\}_{k \in \mathbb{Z}}$, where $v_{k}=i e^{-i k \theta} \frac{d}{d \theta}$; and the relations are

$$
\left[v_{k}, v_{m}\right]=(m-k) v_{k+m} .
$$

Suppose that we have a projective representation of $\mathscr{V}$. If $V_{k}$ is the operator representing $v_{k}$ then

$$
\left[V_{k}, V_{m}\right]=(m-k) V_{k+m}+c\left(v_{k}, v_{m}\right),
$$

where $c: \mathscr{V}_{\mathbb{C}} \times \mathscr{V}_{\mathbb{C}} \rightarrow \mathbb{C}$ is a cocycle of the Lie algebra. It is easy to calculate $c$ for our representation of $\mathscr{V}$ on $H$, but it is worth noticing that there are few possibilities, in view of the following well-known proposition [2].

Proposition (6.2) $H^{2}(\mathscr{V} ; \mathbb{C}) \cong \mathbb{C}$, with generator $c$, where

$$
c(\xi, \eta)=\frac{1}{24 \pi i} \int_{0}^{2 \pi} \xi^{\prime}(\theta)\left(\eta^{\prime \prime}(\theta)+\eta(\theta)\right) d \theta,
$$

so that

$$
\begin{aligned}
c\left(v_{k}, v_{m}\right) & =\frac{1}{12} m\left(m^{2}-1\right) \text { if } k+m=0 \\
& =0 \text { if } k+m \neq 0 .
\end{aligned}
$$

Proof. If $c: \mathscr{V} \times \mathscr{V} \rightarrow \mathbb{C}$ represents an element of $H^{2}(\mathscr{V} ; \mathbb{C})$ we can assume it is invariant under the group $R$ of rotations of the circle, for averaging $c$ over a compact group of inner automorphisms of $\mathscr{V}$ does not change its cohomology class. But if $c$ is $R$-invariant then $c\left(v_{k}, v_{m}\right)=0$ when $k+m \neq 0$, and we have only to determine $c_{k}=c\left(v_{-k}, v_{k}\right)$. From the cocycle condition

$$
c([\xi, \eta], \zeta)+c([\eta, \zeta], \xi)+c([\zeta, \xi], \eta)=0
$$

we find (taking $\xi=v_{k}, \eta=v_{m}, \zeta=v_{-k-m}$, and noticing that $c_{-n}=-c_{n}$ )

$$
(k-m) c_{k+m}=(k+2 m) \mathcal{c}_{k}-(2 k+m) c_{m} .
$$

The most general solution of this is $c_{k}=\lambda k^{3}+\mu k$, with $\lambda, \mu \in \mathbb{C}$. But $c$ can be altered by adding to it a coboundary, i.e. a cocycle of the form $(\xi, \eta) \mapsto f([\xi, \eta])$, where $f: \mathscr{V} \rightarrow \mathbb{C}$ is an $R$-invariant linear map. The only possible $f$ is $f\left(v_{k}\right)=v \delta_{k o}$, for some $v \in \mathbb{C}$; and this changes $c_{k}$ by $2 v k$. The value of $\mu$ is therefore irrelevant. If we normalize $c$ by requiring it to vanish on the Lie algebra of $P S L_{2}(\mathbb{R})$, which is spanned by $\left\{v_{1}, v_{0}, v_{-1}\right\}$ then $c_{k}=\lambda k\left(k^{2}-1\right)$, and we have the result of (6.2).

The cocycle which arises in the representation of $\operatorname{Diff}\left(S^{1}\right)$ on $H$ will be discussed further in Sect 7. It is precisely the cocycle $c$ given in Proposition (6.2), corresponding to $\lambda=\frac{1}{12}$. 
Suppose now that we have a projective unitary representation of $\operatorname{Diff}\left(S^{1}\right)$ on a Hilbert space $K$, corresponding to the cocycle we have been considering. Then $\mathscr{V}_{\mathbb{C}}$ acts on $K$ by the unbounded operators $\left\{V_{k}\right\}$. The group $R$ of rotations has the generator $i V_{0}$, so $e^{2 \pi i V_{0}}=1$, and $V_{0}$ is self-adjoint with integral spectrum. Let $K=\bigoplus K(q)$, where $K(q)$ is the $q$-eigenspace of $V_{0}$. Then $V_{k} \cdot K(q) \subset K(q+k)$. Elements of $K$ which are annihilated by $V_{k}$ for all $k<0$ will be called lowest weight vectors. If the spectrum of $V_{0}$ is bounded below by $q_{0}$ then $K\left(q_{0}\right)$ consists of lowest weight vectors. Using the relations ( $¥)$ we see that if $\Omega \in K\left(q_{0}\right)$ then the cyclic subrepresentations $K_{\Omega}$ of $\operatorname{Diff}\left(S^{1}\right)$ generated by $\Omega$ is the closed subspace of $K$ spanned by the vectors of the form $V_{k_{1}} V_{k_{2}} \ldots V_{k_{r}} \Omega$, where $k_{1} \geqq k_{2} \geqq \ldots \geqq k_{r}>0$. Because $K_{\Omega}\left(q_{0}\right)=\mathbb{C} \cdot \Omega$ it follows that if $K$ is irreducible then $K\left(q_{0}\right)=\mathbb{C} \cdot \Omega$. In fact $K$ is irreducible if and only if it contains no lowest weight vectors other than multiples of $\Omega$. For on the one hand any other lowest weight vector $\Omega^{\prime}$ would be orthogonal to $K_{\Omega}$, and on the other, if $K$ were reducible each piece would contain at least one lowest vector. This means that to decompose $H$ under $\operatorname{Diff}\left(S^{1}\right)$ we have to find all the lowest weight vectors in it. This was done, at least conjecturally, by Goldstone, and his conclusions have been verified by Kač [7].

The lowest weight vectors we have found so far (and in fact there are no others) form the $\mathrm{SU}_{2}$-invariant subspace generated by the vacuum vectors $\left\{\Omega_{2 k}\right\}_{k \in Z}$. In other words, there is a sequence of lowest weight vectors $\Omega_{2 k}^{(m)}$ in $H_{2 k}$, where $\Omega_{2 k}^{(m)}$ has weight $(k+m)^{2}$. (The weight is the eigenvalue of $V_{0}$. ) Up to a scalar multiple $\Omega_{2 k}^{(m)}$ is $\left(B^{*}\right)^{m} \Omega_{2 k+2 m}$ if $k \geqq 0$, and $B^{m} \Omega_{2 k-2 m}$ if $k \leqq 0$. Goldstone has found elegant explicit expressions for the vectors $\Omega_{2 k}^{(m)}$. To explain them let us recall from Sect. 4 the definition of the operators $a_{k}=A\left(e^{i k \theta}\right)$ on $H$. The $a_{k}$ for $k \geqq 1$ commute, and $H_{2 k}$ is a completion of the free cyclic module $\mathbb{C}\left[a_{1}, a_{2}, \ldots\right] \cdot \Omega_{2 k}$ for the polynomial algebra $\mathbb{C}\left[a_{1}, a_{2}, \ldots\right]$. We also introduced operators $c_{1}, c_{2}, \ldots$ related to $a_{1}, a_{2}, \ldots$ by Newton's formulae

$$
\exp \sum_{n>0} \frac{a_{n}}{n} t^{n}=\sum_{n>0} c_{n} t^{n}
$$

i.e.

$$
\begin{aligned}
c_{1} & =a_{1}, \\
2 c_{2} & =a_{1}^{2}+a_{2}, \\
6 c_{3} & =a_{1}^{3}+3 a_{1} a_{2}+2 a_{3}, \text { etc. }
\end{aligned}
$$

(The signs, however, are not the same as in the usual version.) Then $\mathbb{C}\left[a_{1}, a_{2}, \ldots\right]=$ $\mathbb{C}\left[c_{1}, c_{2}, \ldots\right]$; and Goldstone's formulae are

Proposition (6.4) If $k \geqq 0$ then $\Omega_{ \pm 2 k}^{(m)}=f_{2 k}^{(m)} \Omega_{ \pm 2 k}$, up to a scalar multiplier, where

$$
f_{2 k}^{(m)}=\left|\begin{array}{lllll}
c_{2 k+1} & c_{2 k+2} & c_{2 k+3} & \ldots & c_{2 k+m} \\
c_{2 k+2} & c_{2 k+3} & c_{2 k+4} & \ldots & c_{2 k+m+1} \\
\vdots & \vdots & & & \\
c_{2 k+m} & c_{2 k+m+1} & \ldots & \ldots & c_{2 k+2 m-1}
\end{array}\right| .
$$

Proof. We know $\Omega_{-2 k}^{(m)}=B^{m} \Omega_{-2 k-2 m}$. In the notation of Sect. 4 this is

$$
(2 \pi)^{-m} \int \ldots \int B_{\theta_{1}} B_{\theta_{2}} \ldots B_{\theta_{m}} \Omega_{-2 k-2 m} d \theta_{1} \ldots d \theta_{m} .
$$


Now $B_{\theta}=e^{i A_{\theta}^{+}} e^{i A_{\bar{\theta}}} T\left(e^{i q_{\theta}}\right)$. Because

$$
e^{i A_{\bar{\theta}}^{-}} e^{i A_{\phi}^{+}}=\left(1-e^{i(\theta-\phi)}\right)^{2} e^{i A_{\phi}^{+}} e^{i A_{\bar{\theta}}^{-}}
$$

and

$$
T\left(e^{\left.i q_{\theta}\right)} \Omega_{-2 n}=e^{(2 n-1) i \theta} \Omega_{-2 n+1}\right.
$$

we find that the integrand in the above expression is

$$
\Delta\left(\theta_{1}, \ldots, \theta_{m}\right)^{2} e^{i(2 k+1)\left(\theta_{1}+\cdots+\theta_{m}\right)} e^{i A_{\theta}^{+}} \ldots e^{i A_{\theta}^{+} m} \Omega_{-2 k},
$$

where

$$
\Delta\left(\theta_{1}, \ldots, \theta_{m}\right)=\prod_{p<q}\left(e^{i \theta_{p}}-e^{i \theta_{q}}\right) .
$$

Now let us write $z_{k}=e^{i \theta_{k}}$, and observe that $\Delta=\Delta\left(\theta_{1}, \ldots, \theta_{m}\right)$ is the Vandermonde determinant

$$
\left|\begin{array}{ccccc}
1 & z_{1} & z_{1}^{2} & \ldots & z_{1}^{m-1} \\
1 & z_{2} & z_{2}^{2} & \ldots & z_{2}^{m-1} \\
\vdots & & & & \\
1 & z_{m} & z_{m}^{2} & \ldots & z_{m}^{m-1}
\end{array}\right|
$$

Accordingly $\Delta^{2}$ is obtained by summing

$$
\operatorname{sign}(f) \Delta z_{f_{1}}^{m-1} z_{f_{2}}^{m-2} \ldots z_{f_{m}}^{0}=\left|\begin{array}{lllll}
1 & z_{f_{1}} & z_{f_{1}}^{2} & \ldots & z_{f_{1}}^{m-1} \\
z_{f_{2}} & z_{f_{2}}^{2} & z_{f_{2}}^{3} & \ldots & z_{f_{2}}^{m} \\
\vdots & & & & \\
z_{f_{m}}^{m-1} & z_{f_{m}}^{m} & & \ldots & z_{f_{m}}^{2 m-2}
\end{array}\right|
$$

over all permutations $f=\left(f_{1}, f_{2}, \ldots, f_{m}\right)$ of $(1,2, \ldots, m)$. So, because the integrand is symmetric in $\left(\theta_{1}, \ldots, \theta_{m}\right)$ we have

$$
B^{m} \Omega_{-2 k-2 m}=m !(2 \pi)^{-m} \int \ldots \int J\left(z_{1}, \ldots, z_{m}\right) e^{i A_{\theta_{1}^{+}}} \ldots e^{i A_{\theta_{m}^{+}}^{+} \Omega_{-2 k}} d \theta_{1} \ldots d \theta_{m},
$$

where

$$
J\left(z_{1}, \ldots, z_{m}\right)=\left|\begin{array}{llll}
z_{1}^{2 k+1} & z_{1}^{2 k+2} & \ldots & z_{1}^{2 k+m} \\
z_{2}^{2 k+2} & z_{2}^{2 k+3} & \cdots & z_{2}^{2 k+m+1} \\
\vdots & & & \\
z_{m}^{2 k+m} & z_{m}^{2 k+m+1} & \ldots & z_{m}^{2 k+2 m-1}
\end{array}\right| .
$$

This yield the desired formula, because

$$
e^{i A_{\theta_{1}}^{+}} \ldots e^{i A_{\theta_{m}}^{+} \Omega_{-2 k}}=\sum_{k_{1}, \ldots, k_{m}} e^{-i\left(k_{1} \theta_{1}+\ldots+k_{m} \theta_{m}\right)} c_{k_{1}} c_{k_{2}} \ldots c_{k_{m}} \Omega_{-2 k} .
$$

The case of $\Omega_{2 k}^{(m)}$ is precisely similar.

The last result to be proved in this section is

Proposition (6.5) The representation $P_{0}$ is irreducible. 
Proof. It is enough to show that $P_{0}$ is cyclic with respect to its vacuum vector $\Omega_{0}$, for we have seen that if $P_{0}$ contains another lowest weight vector $\Omega^{\prime}$ then $\Omega^{\prime}$ is perpendicular to the cyclic representation generated by $\Omega_{0}$.

Recall from Sect. 5 that an element $T: V \rightarrow V$ of the complexified Lie algebra of $S p_{0}(V)$ can be written

$$
\mathrm{T}=\left(\begin{array}{rr}
\alpha & \beta \\
\bar{\gamma} & -\alpha^{t}
\end{array}\right)
$$

with respect to the decomposition $V_{\mathbb{C}}=W \oplus \bar{W}$. Here $\alpha$ is an endomorphism of $W$, and $\beta$ and $\gamma$ are Hilbert-Schmidt operators $\bar{W} \rightarrow W$ which can be regarded as elements of $\hat{S}^{2}(W)$. The action of $T$ on $\hat{S}(W)$ is $\frac{1}{2} M_{\beta}+D_{\alpha}-\frac{1}{2} M_{\gamma^{\prime}}^{*}$ where $M_{\beta}$ is multiplication by $\beta \in s^{2}(W)$, and $D_{\alpha}$ is the derivation of $S(W)$ induced by $\alpha: W \rightarrow W$. If $T$ is the action of the vector field $v_{k}$, with $k>0$, then it is easy to check that the corresponding $\beta \in \hat{S}^{2}(W)$ is $\beta_{k}=\frac{1}{4} \sum_{i=1}^{k-1} a_{i} a_{k-i}$ in terms of the basis $\left\{a_{m}=e^{\mathrm{im} \theta}\right\}$ of $W$.

Now the Poincaré series of $P_{0}$ is $\prod_{k>1}\left(1-t^{k}\right)^{-1}$. So for dimensional reasons it will certainly be cyclic if the vectors $V_{k_{1}}^{k>1} V_{k_{2}} \ldots V_{k_{r}} \Omega_{0}$ in $H_{0}=\hat{S}(W)$ are all linearly independent when $k_{1} \geqq k_{2} \geqq \ldots \geqq k_{r}>1$. But

$$
V_{k_{1}} V_{k_{2}} \ldots V_{k_{r}} \Omega_{0}=\beta_{k_{1}} \beta_{k_{2}} \ldots \beta_{k_{r}} \Omega_{0}+\text { (terms of lower degree). }
$$

These vectors will be linearly independent if the elements $\beta_{2}, \beta_{3}, \beta_{4}, \ldots$ of the polynomial algebra $S(W)$ are algebraically independent. But that is obvious, for if $\beta_{k}$ were algebraic cover $\mathbb{C}\left[\beta_{2}, \ldots, \beta_{k-1}\right]$ then it would be algebraic over $\mathbb{C}\left[a_{1}, \ldots, a_{k-2}\right]$, and so $a_{k-1}$ would be algebraic over $\mathbb{C}\left[a_{1}, \ldots, a_{k-2}\right]$, which is absurd.

To conclude this section I should remark that it is easy to see that for each $\lambda \in \mathbb{R}$ the Lie algebra $\mathscr{V}$ has a unique irreducible projective representation $P_{\lambda}$ (with the correct cocycle) generated by a lowest weight vector $\omega_{\lambda}$ such that $V_{0} \omega_{\lambda}=\lambda \omega_{\lambda}$. If this is to come from the extension of $\operatorname{Diff}\left(S^{1}\right)$ we are considering then $\lambda$ must be an integer. If the representation is to be unitary then $\lambda$ must not be negative, for otherwise

$$
\left\langle V_{1} \omega_{\lambda}, V_{1} \omega_{\lambda}\right\rangle=\left\langle\omega_{\lambda}, V_{-1} V_{1} \omega_{\lambda}\right\rangle=2\left\langle\omega_{\lambda}, V_{0} \omega_{\lambda}\right\rangle=2 \lambda\left\langle\omega_{\lambda}, \omega_{\lambda}\right\rangle
$$

would be negative. (But of course if $\lambda \geqq 0$ then $\bar{P}_{\lambda}$ is a representation of negative energy with a highest weight $-\lambda$.) If $\lambda$ is not a square Kač has shown that the Poincaré series of $P_{\lambda}$ is $\chi_{\lambda}(t)=t^{\lambda} \pi(t)$. But when $\lambda=q^{2}$ the representation $P_{\lambda}$ suddenly becomes smaller, and its Poincaré series is

$$
\chi_{q^{2}}(t)=t^{q^{2}}\left(1-t^{2 q+1}\right) \pi(t) .
$$

Notice that $t^{q^{2}} \pi(t)=\sum_{r \geqq q} \chi_{r^{2}}(t)$.

The Decomposition of the Representation of $\operatorname{Map}\left(S^{1} ; G\right)$ for other $G$

Let us now turn to the more general situation of the last part of Sect. 4. We considered there some irreducible projective representations of $M=\operatorname{Map}\left(S^{1}: T\right)$, where $T$ was a torus. The representations are of the form

$$
H=\underset{\lambda \in X}{\oplus} H_{\lambda},
$$


where $H_{\lambda}$ is an irreducible representation of the identity component $M_{0}$ of $M$ on which $T \subset M$ acts by the character $\lambda$. The set $X$ of characters is a coset of $L=\pi_{1}(T)$ in $L^{*}=\operatorname{Hom}(T ; \pi)$.

The group $\operatorname{Diff}\left(S^{1}\right)$ is a group of automorphisms of the central extension $\hat{M}$ of $M$ : it does not preserve the cocycle $c$ defining $\hat{M}$, but acts by

$$
\phi^{*}\left(z, e^{i f}\right)=z e^{-(1 / 2) i\left\langle\Delta_{f}, f(\phi(0))-f(0)\right\rangle}, \phi^{*}\left(e^{i f}\right) .
$$

This means that $\operatorname{Diff}\left(S^{1}\right)$ acts projectively on $H$, as before, preserving each subspace $H_{\lambda}$. To find the cocycle which arises it is enough to consider $H_{0}$, which is an irreducible representation of the vector space $V=M_{0} / T$. This vector space, with its skew form, is simply the product of $d$ copies of $V^{(1)}=\operatorname{Map}\left(S^{1} ; \mathbb{R}\right) / \mathbb{R}$, where $d$ is the dimension of $T$. $H_{0}$ is therefore the tensor product of $d$ copies of $H_{0}^{(1)}$, the irreducible representation of $V^{(1)}$. This gives us

Proposition (6.6) $\operatorname{Diff}\left(S^{1}\right)$ acts projectively with the cocycle d.c on the faithful irreducible representations of $\operatorname{Map}\left(S^{1} ; T\right)$, where $d=\operatorname{dim}(T)$, and $c$ is cocycle of Proposition (6.2).

By considering the action of the Lie algebra $\mathscr{V}$ on $H_{0}$ we know a priori that under $\operatorname{Diff}\left(S^{1}\right)$ the decomposition of $H_{0}$ must be of the form

$$
H_{0}=\underset{q}{\oplus}\left(P_{q}^{d}\right)^{m_{q}}
$$

where $P_{q}^{d}$ is an irreducible representation of $\mathscr{V}$ with the cocycle $d . c$ generated by a lowest weight vector with positive integral lowest weight $q$. (It is clear from general considerations that up to isomorphism there can be only one such representation $P_{q}^{d}$.) The case $d>1$ is slightly easier than the one we have already studied, and we have

Proposition (6.7) The Poincaré series of $P_{q}^{d}$ is $t^{q} \pi(t)$ if $q>0$, and is $(1-t) \pi(t)$ if $q=0$. Proof. When $q=0$ the proof is essentially the same as that of (6.5). $P_{q}^{d}$ can be identified with the cyclic $\mathscr{V}$-module generated by the vacuum vector in $H_{0}^{q}=\hat{S}(W)$. Choose an orthonormal basis $\left\{\xi_{1}, \ldots, \xi_{d}\right\}$ of the Lie algebra $t$ of $T$. This gives one a basis $\left\{a_{M}^{j}=\xi^{j} e^{\mathrm{im} \theta}\right\}(\mathrm{j}=1, \ldots, d ; m=1,2,3, \ldots)$ of $W$. The elements $\beta_{k}$ of $(6.5)$ are replaced by

$$
\beta_{k}^{(d)}=\frac{1}{2} \sum_{i=1}^{k-1} \sum_{j=1}^{d} a_{i}^{j} a_{k-j}^{j},
$$

and $\beta_{2}^{(d)}, \beta_{3}^{(d)}, \ldots$ are algebraically independent as before.

To treat $P_{q}^{d}$ for $q>0$ we consider the action of $\mathscr{V}$ on $H_{\lambda}$. The vacuum vector $\Omega_{\lambda}$ of $H_{\lambda}$ is obtained from $\Omega_{0}$ by the action of $e^{i \lambda} \in M$, so the argument we have used earlier when $d=1$ shows that the group of rotations $R$ acts on $\Omega_{\lambda}$ with weight $\frac{1}{2}\langle\lambda, \lambda\rangle$. We choose $\lambda$ so that $\frac{1}{2}\langle\lambda, \lambda\rangle=q$ and let $P_{q}^{d}$ be the $\mathscr{V}$-module generated by $\Omega_{\lambda}$. We can calculate the action $\tilde{V}_{k}$ of $v_{k} \in V$ on $H_{\lambda}$ in terms of its action $V_{k}$ on $H_{0}$ : when $H_{0}$ and $H_{\lambda}$ are both identified with $\hat{S}(W)$ we have

$$
\tilde{V}_{k}=V_{k}+\sum_{j} \lambda_{j} a_{k}^{j}
$$

when $k \neq 0$, where $\left\{\lambda_{j}\right\}$ are the components of $\lambda$ with respect to the basis of $t$. In 
other words, when $k>0$ the action of $\tilde{V}_{k}$ on $\widehat{S}^{r}(W)$ is multiplication by $\widetilde{\beta}_{k}^{(d)}=$ $\beta_{k}^{(d)}+\sum \lambda_{j} a_{k}^{j}$ modulo $\hat{S}^{r}(W)$. The elements $\widetilde{\beta}_{k}^{(d)}$ for $k=1,2,3, \ldots$ are algebraically independent, and that establishes our result.

In view of (6.7) we can write down at once the decomposition of $H_{0}$ under $\operatorname{Diff}\left(S^{1}\right)$ : the result is that $P_{q}^{d}$ occurs with multiplicity $m_{q}$ equal to the coefficient of $t^{q}$ in $t+\pi(t)^{d-1}$.

It is more interesting, however, to decompose the space $H=\oplus H_{\lambda}$ under the group $G \times \operatorname{Diff}\left(S^{1}\right)$. To do so we write down the character of $H$ as a representation $T \times R$. It is

$$
F=\pi(t)^{d} \sum_{\lambda \in L} e^{i \lambda} t^{1 / 2\langle\lambda, \lambda\rangle}
$$

where $t$ is now regarded as the "identity" character $R \rightarrow \mathbb{T}$. We should like to write this in the form $\sum_{\mu \in P} f_{\mu}(t) \chi_{\mu}$, where $P$ is the set of dominant weights in $L$, and $\chi_{\mu}$ is the character of the irreducible representation of $G$ associated to $\mu \in P$. The following result was shown to me by Macdonald. Proposition (6.8) The character of $H$ as a representation of $G \times R$ is $\sum_{\mu \in P} f_{\mu}(t) \chi_{\mu}$,
where

$$
f_{\mu}(t)=\pi(t)^{d} t^{(1 / 2)\langle\mu, \mu\rangle} \prod_{\alpha>0}\left(1-t^{\langle\alpha, \mu+\rho\rangle}\right) .
$$

(Here the product is over the positive roots of $G$, and $\rho$ is half the sum of the positive roots.)

Proof. By the Weyl character formula $f_{\mu}(t)$ is the coefficient of $e^{i(\mu+\rho)}$ in $\Delta \cdot F$, where $\Delta=\sum_{w \in W}(-1)^{w} e^{i w \rho}$ is the Weyl denominator, $W$ being the Weyl group of $G$. This gives

$$
f_{\mu}(t)=\pi(t)^{d} \sum_{w \in W}(-1)^{w} t^{(1 / 2)\langle\mu+\rho-w \rho, \mu+\rho-w \rho\rangle} .
$$

But we know from the Weyl denominator identity that

$$
\sum_{w \in W}(-1)^{w} t^{\langle w \rho, \xi\rangle}=\prod_{\alpha>0}\left(t^{(1 / 2)\langle\alpha, \xi\rangle}-t^{-(1 / 2)\langle\alpha, \xi\rangle}\right)
$$

for any $\xi \in L$; and applying that when $\xi=-\mu-\rho$ gives us the desired formula.

The character of $H$ can also be calculated in a completely different way by using the generalized Weyl character formula of Kač[6]. I shall not give the details here, but the result is

where $\Pi=\pi(t)^{d} \prod_{k=1}^{\infty} \prod_{\alpha}\left(1-t^{k} e^{i \alpha}\right), \alpha$ runs over all the roots of $G$, and $n$ is the Coxeter number of $G$. Equating this to our earlier expression gives the interesting identity

$$
\sum_{\lambda \in L} \chi_{n \lambda} t^{(1 / 2) n\langle\lambda, \lambda\rangle+\langle\lambda, \rho\rangle}=\sum_{\lambda \in L} e^{i \lambda} t^{(1 / 2)\langle\lambda, \lambda\rangle} \prod_{k=1}^{\infty} \prod_{\alpha}\left(1-t^{k} e^{i \alpha}\right),
$$

which has also been found by Kač from another point of view ([6](3.38)). 


\section{Group Extensions}

(a) $\operatorname{Map}\left(S^{1} ; G\right)$

In Sect. 4 we constructed some projective unitary representations of the group $\Gamma=\operatorname{Map}\left(S^{1} ; G\right)$, where $G$ was a compact, simply connected, simply laced Lie group, but we did not describe explicitly the central extension $\mathbb{T} \rightarrow \hat{\Gamma} \rightarrow \Gamma$ involved. We shall now discuss it further.

The first point to notice is that as a topological space $\hat{\Gamma}$ is not the product of $\Gamma$ and $\mathbb{T}$, so it cannot be described by a continuous cocycle $\Gamma \times \Gamma \rightarrow \mathbb{T}$ as in Sect. 2 . (This is closely related to the fact that $\hat{\Gamma}$ does not come from an extension of $\Gamma$ by $\mathbb{R}$. It is easy to see that if an extension of a group $\Gamma$ by $\mathbb{T}$ lifts to $\mathbb{R}$ then it is topologically a product. The converse is true if $\Gamma$ is connected, but not otherwise: the extension $\hat{M} \rightarrow M$ of Sect. 2 is topologically a product but does not lift to $\mathbb{R}$.)

The topological type of the circle bundle $\hat{\Gamma} \rightarrow \Gamma$ can be determined from the extension of Lie algebras

$$
\mathbb{R} \rightarrow \operatorname{Lie}(\hat{\Gamma}) \rightarrow \operatorname{Lie}(\Gamma)
$$

in view of the following simple result, which I think is well-known.

Proposition (7.1) If a group extension $\mathbb{T} \rightarrow \hat{\Gamma} \rightarrow \Gamma$ corresponds to the Lie algebra cohomology class $\omega \in H^{2}(\operatorname{Lie}(\Gamma) ; \mathbb{R})$, then the image of $\frac{\omega}{2 \pi}$ under the map

$$
H^{2}(\operatorname{Lie}(\Gamma) ; \mathbb{R}) \rightarrow H^{2}(\Gamma ; \mathbb{R})
$$

is the first Chern class of the circle-bundle $\hat{\Gamma} \rightarrow \Gamma$ (with real coefficients).

The map in (7.1) is the one which interprets a skew multilinear form on Lie $(\Gamma)$ as a left-invariant differential form on the manifold $\Gamma$. (The $2 \pi$ comes in because we are identifying $\mathbb{T}$ with $\mathbb{R} / 2 \pi \mathbb{Z}$.)

Let us recall from Sect. 4 that in our case the extension of Lie algebras is defined by the cocycle $\omega$, where

$$
\omega(\phi, \psi)=\frac{1}{2 \pi} \int_{0}^{2 \pi}\left\langle\phi^{\prime}(\theta), \psi(\theta)\right\rangle d \theta,
$$

and $\langle$,$\rangle is the invariant inner product on \mathfrak{g}=\operatorname{Lie}(G)$ described in Sect. 4 .

For any compact Lie group $G$ we can define elements $\lambda$ in $H^{2}(\Gamma ; \mathbb{Z})$, and hence natural circle-bundles on $\Gamma$, by the transgression of elements $\alpha \in H^{3}(G ; \mathbb{Z})$ : one pulls $\alpha$ back to $\varepsilon^{*}(\alpha) \in H^{3}\left(S^{1} \times \Gamma\right)$ by the evaluation map $\varepsilon: S^{1} \times \Gamma \rightarrow G$, and integrates over $S^{1}$ to get $\lambda \in H^{2}(\Gamma)$.

With real coefficients $\alpha$ can be represented by a left-invariant form, again denoted $\alpha$, given by

$$
\alpha(\xi, \eta, \zeta)=\frac{1}{8 \pi^{2}}\langle[\xi, \eta], \zeta\rangle,
$$

where $\xi, \eta, \zeta \in \mathfrak{g}$ are thought of as tangent vectors to $G$ at some point, and $\langle$,$\rangle is$ an invariant inner product on $\mathfrak{g}$. We have

Proposition (7.2) If $G$ is simply connected then $\alpha$ is an integral class if and only if $\langle\lambda, \lambda\rangle \in 2 \mathbb{Z}$ for each co-root $\lambda$ in the Lie algebra of the maximal torus of $G$. 
Let us recall that the definition of co-roots does not involve choosing an inner product on $\mathfrak{g}$ : in particular, if $\lambda_{\alpha}$ is the co-root associated to a root $\alpha$ then $\alpha\left(\lambda_{\alpha}\right)=2$. The condition of (7.2) is satisfied for the inner product of Sect. 4.

Now suppose $\phi, \psi: S^{1} \rightarrow \mathfrak{g}$ are two tangent vectors to $\Gamma$ at $f: S^{1} \rightarrow G$. If $\phi, \psi$ and $\frac{d}{d \theta}$ are regarded as tangent vectors to $S^{1} \times \Gamma$ at $(\theta, f)$ then

$$
\varepsilon^{*} \alpha_{(\theta, f)}\left(\phi, \psi, \frac{d}{d \theta}\right)=\left\langle[\phi(\theta), \psi(\theta)], f(\theta)^{-1} f^{\prime}(\theta)\right\rangle,
$$

and accordingly $\lambda$ is given at $f \in \Gamma$ by

$$
\lambda_{f}(\phi, \psi)=\frac{1}{8 \pi^{2}} \int_{0}^{2 \pi}\left\langle[\phi(\theta), \psi(\theta)], f(\theta)^{-1} f^{\prime}(\theta)\right\rangle d \theta .
$$

This is not an invariant differential form on $\Gamma$, but it is cohomologous to $\frac{\omega}{2 \pi}$, where $\omega$ is the invariant form defined above. In fact $\lambda=\frac{\omega}{2 \pi}+d \beta$, where

$$
\beta_{f}(\phi)=\frac{1}{8 \pi^{2}} \int_{0}^{2 \pi}\left\langle\phi(\theta), f(\theta)^{-1} f^{\prime}(\theta)\right\rangle d \theta .
$$

If $G$ is connected and simply connected then so is $\Gamma$, because $\pi_{2}(G)=0$. In that case $H^{2}(\Gamma ; \mathbb{Z}) \rightarrow H^{2}(\Gamma ; \mathbb{R})$ is injective, and we have

Proposition (7.3) If $\mathbb{T} \rightarrow \hat{\Gamma} \rightarrow \Gamma$ is a group extension corresponding to the Lie algebra cocycle $\omega$ then topologically $\hat{\Gamma}$ is the circle-bundle on $\Gamma$ with Chern class $\lambda \in H^{2}(\Gamma ; \mathbb{Z})$.

We have still not proved the existence of any such extension $\hat{\Gamma}$, except by the indirect method of Sect. 4. One way to remedy this is by analogy with the discussion in Sect. 5.

Let $M$ be a finite dimensional real representation of $G$ with an inner product $\langle$,$\rangle , and let V$ denote the vector space of smooth maps $S^{1} \rightarrow M$ modulo constants. $V$ has an inner product given by

$$
\langle\phi, \psi\rangle=\frac{1}{2 \pi} \int_{0}^{2 \pi}\langle\xi(\theta), \eta(\theta)\rangle d \theta
$$

The group $\Gamma$ acts orthogonally on $V$. As in Sect. 5 we decompose the complexification of $V$ as $V_{\mathbb{C}}=W \oplus \bar{W}$, where $W$ is the holomorphic maps of the unit disk into $M$ (modulo constants). If $f$ is an orthogonal transformation of $V$ we write it as a matrix

$$
\left(\begin{array}{ll}
a(f) & b(f) \\
\overline{b(f)} & \overline{a(f)}
\end{array}\right)
$$

with respect to the decomposition $V_{\mathbb{C}}=W \oplus \bar{W}$.

In the symplectic case the operator $a(f): W \rightarrow W$ was necessarily invertible. This is no longer true in the present situation, but nevertheless for any $f \in \Gamma$ it is 
easy to see that $a(f)$ is a Fredholm operator, and $b(f)$ is Hilbert-Schmidt. It follows that $f \mapsto a(f)$ defines a homomorphism $\Gamma \rightarrow \operatorname{Aut}(W) / \operatorname{Aut}_{1}(W)$, where $\operatorname{Aut}_{1}(W)$ are the automorphisms of $W$ of the form (identity) + (operator of trace class). Thus the extension

$$
\operatorname{Aut}_{1}(W) \rightarrow \operatorname{Aut}(W) \rightarrow \operatorname{Aut}(W) / \operatorname{Aut}_{1}(W)
$$

pulls back to define an extension of $\Gamma$ by $\operatorname{Aut}_{1}(W)$. But on $\operatorname{Aut}_{1}(W)$ we have the determinant, a homomorphism

$$
\text { det : } \operatorname{Aut}_{1}(W) \rightarrow \mathbb{C}^{\times},
$$

and this gives us an extension $\tilde{\Gamma}$ of $\Gamma$ by $\mathbb{C}^{\times}$.

Proposition (7.4) The extension $\mathbb{C}^{\times} \rightarrow \tilde{\Gamma} \rightarrow \Gamma$ corresponds to the Lie algebra cocycle $\tilde{\omega}$ defined by

$$
\tilde{\omega}(\phi, \psi)=\frac{1}{2 \pi} \int_{0}^{2 \pi}\left\langle\phi^{\prime}(\theta), \psi(\theta)\right\rangle_{M} d \theta,
$$

where $\langle,\rangle_{M}$ is the trace-form of $M\left(\right.$ i.e. $\langle\xi, \eta\rangle_{M}=\operatorname{trace}\left(\xi_{M} \eta_{M}\right)$, where $\xi_{M}$ is the action of $\xi \in \mathfrak{g}$ on $M$ ).

Proof. Let the derivative of $f \mapsto a(f)$ at the identity be denoted by $\phi \mapsto A(\phi)$. We have to show that

$$
\text { trace }\{[A(\phi), A(\psi)]-A([\phi, \psi])\}=-4 i \tilde{\omega}(\phi, \psi)
$$

for $\phi, \psi: S^{1} \rightarrow \mathfrak{g}$.

We can write

$$
\operatorname{Lie}(\Gamma)=\bigoplus_{k=-\infty}^{\infty} L_{k} \text { and } W=\bigoplus_{k=1}^{\infty} W_{k^{\prime}}
$$

where $L_{k}=\mathfrak{g} \cdot z^{k}$ and $W_{k}=M_{\mathbb{C}} \cdot z^{k}$. Obviously $\left[L_{k}, L_{m}\right] \subset L_{k+m}$, and if $\phi \in L_{k}$ then $A(\phi) \cdot W_{k} \subset W_{k+m}$, where $W_{n}=0$ if $n \leqq 0$. It is enough to prove (*) for homogeneous elements $\phi \in L_{k}$ and $\psi \in L_{m}$. If $k+m \neq 0$ then the left-hand side is zero because the matrices of $[A(\phi), A(\psi)]$ and $A([\phi, \psi])$ have no diagonal entries; and $\tilde{\omega}(\phi, \psi)=0$ also. If $\phi=\xi z^{k} \in L_{k}$ with $\xi \in \mathfrak{g}$ and $k \geqq 0$, and $\psi=\eta z^{-k} \in L_{-k}$ then $[A(\phi), A(\psi)]$ and $A([\phi, \psi])$ both preserve each subspace $W_{m}$ and coincide on $W_{m}$ if $m>k$. If $m \leqq k$ then

$$
\begin{aligned}
\{[A(\phi), A(\psi)]-A([\phi, \psi])\} \cdot \zeta z^{m} & =-\left(\eta_{M} \xi_{M} \zeta+[\xi, \eta]_{M} \zeta\right) z^{m} \\
& =\xi_{M} \eta_{M} \zeta z^{m} .
\end{aligned}
$$

so the trace on $W_{m}$ is $\langle\xi, \eta\rangle_{M}$. The trace on all of $W$ is accordingly

$$
\begin{aligned}
k\langle\xi, \eta\rangle_{M} & =\frac{1}{2 \pi i} \int\left\langle\xi z^{k} \cdot d\left(\eta z^{-k}\right)\right\rangle \\
& =-4 i \tilde{\omega}(\phi, \psi),
\end{aligned}
$$

in accordance with $\left(^{*}\right)$.

The extension $\hat{\Gamma}$ which we want can now be constructed. Choose a real orthogonal representation $M$ of $G$ such that $\langle\xi, \eta\rangle_{M}=n\langle\xi, \eta\rangle$, where $n$ is an integer. 
Then $\hat{\Gamma}$ is an $n$-fold covering of $\tilde{\Gamma}:$ in fact $\pi_{1}(\tilde{\Gamma})=\mathbb{Z} / n$, and $\hat{\Gamma}$ is the universal covering of $\tilde{\Gamma}$. In the case of simple simply laced groups one can take for $M$ the adjoint representation of $G$ : then $n$ is the Coxeter number of $G$.

Remark. The Infinite Dimensional Spin Group

One could carry the preceding discussion further in precise analogy with Sect. 5. One can define a subgroup $O_{0}(V)$ of the orthogonal group $O(V)$, and a central extension

$$
\mathbb{T} \rightarrow \operatorname{Spin}_{0}^{c}(V) \rightarrow O_{0}(V) .
$$

$\operatorname{Spin}_{0}^{c}(V)$ acts unitarily on the completion of the exterior algebra $\Lambda(W)$ : this is the "spin" representation [11], analogous to the metaplectic representation of $S p_{0}(V)$. It can be constructed by starting with a space $X$ of polarizations of $V$ such that $X \cong O_{0}(V) / U(W)$, defining a holomorphic line bundle $L$ on $X$ whose fibre at $U$ (where $V_{\mathbb{C}}=U \oplus \bar{U}$ ) is $\operatorname{det}(U)^{1 / 2}$, observing that $\operatorname{Spin}_{0}^{c}(V)$ acts on $L$, and associating to each fibre of $L$ a ray in $\hat{\Lambda}(W)$. The only significant difference between this and the symplectic case is that $L$ is now not a trivial bundle. One might have guessed that $\pi_{0}\left(O_{0}(V)\right)=\mathbb{Z}$ because any $f \in O_{0}(V)$ defines a Fredholm operator $a(f): W \rightarrow W$ which has an index in $\mathbb{Z}$; but it turns out that $a(f)$ must have index zero, because $w \mapsto b(f) \bar{w}$ is an isomorphism $\operatorname{ker} a(f) \rightarrow \operatorname{ker} a(f)^{*}$; and $\pi_{0}\left(O_{0}(V)\right)=$ $\mathbb{Z} / 2$, with the components distinguished by the parity of the dimension of $\operatorname{ker} a(f)$.

(b) $\operatorname{Diff}\left(S^{1}\right)$

We have already seen how a central extension of $\operatorname{Diff}\left(S^{1}\right)$ by $\mathbb{T}$ arises from the embedding $\operatorname{Diff}\left(S^{1}\right) \rightarrow S p_{0}(V)$, where $V=\operatorname{Map}\left(S^{1} ; \mathbb{R}\right) /($ constants). This extension is topologically a product, so it can be defined by a cocycle $\omega: \operatorname{Diff}\left(S^{1}\right) \times \operatorname{Diff}\left(S^{1}\right)$ $\rightarrow \mathbb{T}$. Indeed we know that

$$
\omega(\phi, \psi)=\mu \operatorname{det}\left(a(\phi)^{-1} a(\phi \psi) a(\psi)^{-1}\right)^{-1 / 2},
$$

where $a(\phi): W \rightarrow W$ is the component of $\phi: V \rightarrow V$ in the decomposition $V_{\mathbb{C}}=W \oplus \bar{W}$, and $\mu: \mathbb{C}^{\times} \rightarrow \mathbb{T}$ is the projection. We should like, however, to have a more explicit formula for the cocycle.

Such a formula has been found by Bott. If $\phi: S^{1} \rightarrow S^{1}$ is a diffeomorphism (where $S^{1}$ is regarded as $\mathbb{R} / 2 \pi \mathbb{Z}$ ) define $\hat{\phi}: \mathbb{T} \rightarrow \mathbb{T}$ (where $\mathbb{T}=\{z \in C:|z|=1\}$ ) by $\phi\left(e^{i \theta}\right)=e^{i \phi(\theta)}$. Then Bott's cocycle $\tilde{\omega}$ is given by

$$
\tilde{\omega}(\phi, \psi)=e^{i w(\phi, \psi)}
$$

where

$$
w(\phi, \psi)=\operatorname{Re} \int_{S^{1}} \log \phi^{\prime} \cdot d \log \chi^{\prime},
$$

and $\chi=\psi \phi$. (Here $\phi^{\prime}\left(e^{i \theta}\right)$ is to be interpreted as $\phi^{\prime}(\theta) e^{i(\phi(\theta)-\theta)}$.)

I shall show that the cocycles $\omega$ and $\tilde{\omega}$ are cohomologous, i.e. that they define the same extension of $\operatorname{Diff}\left(S^{1}\right)$ by $\mathbb{T}$. I do not know whether they are actually equal: it seems to me an interesting question.

Before comparing $\omega$ and $\tilde{\omega}$ we again need some general remarks. A group extension $\mathbb{T} \rightarrow \widehat{G} \rightarrow G$ of the kind we are considering is a smooth principal $\mathbb{T}$ bundle on the manifold $G$. If one chooses a vector space splitting of the extension 
of Lie algebras $\mathbb{R} \rightarrow \hat{\mathfrak{g}} \rightarrow \mathfrak{g}$ then the elements of $\mathfrak{g}$ define left-invariant vector fields on $\hat{G}$, and hence a left-invariant connection in the $\mathbb{T}$-bundle. The curvature of this connection is a left-invariant 2 -form $\alpha$ on $G$. It is easy to check that $\alpha$ represents the Lie algebra cohomology class of the extension $\hat{\mathrm{g}}$ : it is the representative defined by the chosen splitting $\hat{\mathfrak{g}} \cong \mathfrak{g} \oplus \mathbb{R}$.

The class $\alpha \in H^{2}(\mathfrak{g} ; \mathbb{R})$ nearly determines the extension $\hat{G}$. To see that, let us recall that the extensions of $G$ by $\mathbb{T}$ form an abelian $\operatorname{group} \operatorname{Ext}(G ; \mathbb{T})$. One way to obtain an extension is to take a homomorphism $\theta: \pi_{1}(G) \rightarrow \mathbb{T}$ and to define $\widehat{G}$ as the extension $(\widetilde{G} \times \mathbb{T}) / \pi_{1}(G)$ induced by $\theta$ from the universal covering $\widetilde{G} \rightarrow G$. This gives a homomorphism

$$
\operatorname{Hom}\left(\pi_{1}(G) ; \mathbb{T}\right) \rightarrow \operatorname{Ext}(G ; \mathbb{T}) \text {. }
$$

the extensions so obtained are flat vector bundles, and we have

Proposition (7.4) The sequence

$$
\operatorname{Hom}\left(\pi_{1}(G) ; \mathbb{T}\right) \rightarrow \operatorname{Ext}(G ; \mathbb{T}) \rightarrow H^{2}(\mathfrak{g} ; \mathbb{R}) \rightarrow H^{2}(G ; \mathbb{T})
$$

is exact. (The right-hand map is the composite of $H^{2}(\mathfrak{g} ; \mathbb{R}) \rightarrow H^{2}(G ; \mathbb{R})$, which regards a left-invariant form as a de Rham cohomology class, and $H^{2}(G ; \mathbb{R}) \rightarrow H^{2}(G ; \mathbb{T})$.)

I shall not give a proof of this here: but exactness at $\operatorname{Ext}(G ; \mathbb{T})$, which is what we shall use, holds because if $\alpha \in H^{2}(\mathfrak{g} ; \mathbb{R})$ is zero then the splitting $\hat{\mathfrak{g}} \cong \mathfrak{g} \oplus \mathbb{R}$ can be chosen so that the connection in the circle-bundle $\widehat{G} \rightarrow G$ is flat, and so defines a homomorphism $\pi_{1}(G) \rightarrow \mathbb{T}$. The result is of course very well-known when $G$ is finite-dimensional, and I state it here to emphasize that finite-dimensionality is not required. The consequence that is relevant for us is

Corollary (7.5) An extension of $\operatorname{Diff}\left(S^{1}\right)$ by $\mathbb{T}$ is determined by its infinitesimal class in $H^{2}\left(\operatorname{Vect}\left(S^{1}\right) ; \mathbb{R}\right)$ together with its restriction to $\operatorname{PSL}_{2}(\mathbb{R}) \subset \operatorname{Diff}\left(S^{1}\right)$. In fact $\operatorname{Ext}\left(\operatorname{Diff}\left(S^{1}\right) ; \mathbb{T}\right) \cong \mathbb{T} \times \mathbb{R}$.

The corollary follows from $(7.4)$ because $\pi_{1}\left(\operatorname{Diff}\left(S^{1}\right)\right)=\pi_{1}\left(\operatorname{PGL}_{2}(\mathbb{R})\right)=\mathbb{Z}$, and $\operatorname{Hom}\left(\pi_{1}\left(\mathrm{PGL}_{2}(\mathbb{R})\right) ; \mathbb{T}\right) \cong \operatorname{Ext}\left(\mathrm{PGL}_{2}(\mathbb{R}) ; T\right)$ because $\mathrm{PGL}_{2}(\mathbb{R})$ is semisimple. We saw in Sect. 6 that $H^{2}\left(\operatorname{Vect}\left(S^{1}\right) ; \mathbb{R}\right) \cong \mathbb{R}$.

Another way to formulate the result (7.5) is to say that the group $G=\operatorname{Diff}\left(S^{1}\right)$ has a universal central extension $A \rightarrow G$ with $A=\mathbb{Z} \oplus \mathbb{R}$. This $E$ is an extension $\mathbb{R} \rightarrow E \rightarrow \widetilde{G}$, where $\widetilde{G}$ is the simply connected covering of $G$, the group of diffeomorphisms $\phi: \mathbb{R} \rightarrow \mathbb{R}$ satisfying $\phi(\theta+2 \pi)=\phi(\theta)+2 \pi$.

Returning to the cocycles $\omega$ and $\tilde{\omega}$, we observe first that they both vanish identically on $\operatorname{PSL}_{2}(\mathbb{R})$, which is the group of holomorphic automorphisms of the unit disk in $\mathbb{C}$, and is the subgroup of $\operatorname{Diff}\left(S^{1}\right)$ which preserves the polarization $V_{\mathbb{C}}=W \oplus \bar{W}$. In the case of $\omega$ we have only to notice that the restriction of $a: \operatorname{Diff}\left(S^{1}\right) \rightarrow \operatorname{Aut}(W)$ to $\operatorname{PSL}_{2}(\mathbb{R})$ is a homomorphism. In the case of $\tilde{\omega}$ the integral defining $w(\phi, \psi)$ vanishes for $\phi, \psi \in \mathrm{PSL}_{2}(\mathbb{R})$ by Cauchy's theorem, as then $\hat{\phi}$ and $\hat{\chi}$ extend to holomorphic functions in the disk.

Now let us consider the Lie algebra cocycles induced by $\omega$ and $\tilde{\omega}$. The general formula for the Lie algebra cocycle associated to a cocycle $c: G \times G \rightarrow \mathbb{R}$ is

$$
(\xi, \eta) \mapsto D^{2} c(\xi, n)-D^{2} c(n, \xi),
$$

where $D^{2} c: \mathfrak{g} \times \mathfrak{g} \rightarrow \mathbb{R}$ is the mixed second derivative of $c$ at $(1,1)$. 
In the case of $\tilde{\omega}$ an elementary calculation gives

$$
(\xi, \eta) \mapsto \frac{1}{24 \pi} \int_{0}^{2 \pi}\left(\xi^{\prime \prime \prime}(\theta)+\xi^{\prime}(\theta)\right) \eta(\theta) d \theta,
$$

whereas from $\omega$ we get

$$
(\xi, \eta) \mapsto \frac{1}{2} i \operatorname{trace}\left([\xi, \eta]_{W}-\left[\xi_{W}, \eta_{W}\right]\right),
$$

where $\xi_{W}: W \rightarrow W$ denotes the $W-W$ component of the action of $\xi$ on $V$. Each of these formulae is invariant under the group $R$ of rotations, so to prove they coincide it is enough to evaluate them on $\xi=v_{-k}$ and $\eta=v_{k}$. The first formula gives $\frac{i}{12} k\left(k^{2}-1\right)$, so we must show

$$
\operatorname{trace}\left(2 k v_{0, W}-\left[v_{-k, W}, v_{k, W}\right]\right)=\frac{1}{6} k\left(k^{2}-1\right) .
$$

The calculation is just like that in the proof of (7.3). The operators $2 k v_{0, W}$ and $\left[v_{-k, W}, v_{k, W}\right]$ are both diagonal with respect to the basis elements $\left\{a_{m}\right\}_{m \geq 1}$ of $W$. They coincide on $a_{m}$ if $m>k$. If $m \leqq k$ then $2 k v_{0, W}$ multiplies $a_{m}$ by $2 k m$. But

$$
\begin{aligned}
{\left[v_{-k, W}, v_{k, W}\right] a_{m} } & =v_{-k, W} v_{k, W} a_{m} \\
& =m(m+k) a_{m},
\end{aligned}
$$

and so the trace is $\sum_{k=1}^{m} m(k-m)=\frac{1}{6} k\left(k^{2}-1\right)$, as we want.

Before leaving the central extension of $\operatorname{Diff}\left(S^{1}\right)$ it would be pointed that the extension of Lie algebras

$$
\mathbb{R} \rightarrow \operatorname{Vect}\left(S^{1}\right)^{\wedge} \rightarrow \operatorname{Vect}\left(S^{1}\right)
$$

is closely related to the "Schwarzian derivative". One ordinarily thanks of this as a third-order non-linear differential operator $S$ defined on functions on the circle, characterized by the property that

$$
S(a \phi+b / c \phi+d)=S(\phi)
$$

for any constants $a, b, c, d$. The formula is

$$
S(\phi)=\frac{1}{2} \frac{\phi^{\prime \prime \prime}}{\phi^{\prime}}-\frac{3}{4}\left(\frac{\phi^{\prime \prime}}{\phi^{\prime}}\right)^{2}
$$

I shall think of it, however, as defined for diffeomorphisms $\phi$ of $S^{1}=R / 2 \pi Z$ by the formula

$$
\sigma(\phi)=\frac{1}{6} S(\phi)+\frac{1}{24}\left(\left(\phi^{\prime}\right)^{2}-1\right) .
$$

Then it has the properties

(a) $\sigma(\phi \psi)=\sigma(\psi)$ if $\phi \in \operatorname{PSL}_{2}(R)$, and

(b) $\sigma(\phi \psi)=\psi^{*} \sigma(\phi) \cdot\left(\psi^{\prime}\right)^{2}+\sigma(\psi)$.

Here $\psi^{*}$ is the operation defined by $\left(\psi^{*} f\right)(\theta)=f(\psi(\theta))$. The second property can be expressed by saying that $\sigma$ is a crossed homomorphism from $\operatorname{Diff}\left(S^{1}\right)$ to $Q$, the space of quadratic differentials on $S^{1}$. 
New $Q$ is naturally dual to Vect $\left(S^{1}\right)$. To describe an extension of the Lie algebra $\operatorname{Vect}\left(S^{1}\right)$ by $\mathbb{R}$ it certainly suffices to give the adjoint action of $\operatorname{Diff}\left(S^{1}\right)$ on $\mathbb{R} \oplus \operatorname{Vect}\left(S^{1}\right)$, or dually on $\mathbb{R} \oplus Q$. The latter action preserves the affine hyperplane $1 \oplus Q$, and is obviously determined by its restriction to that. This restriction can be thought of as an affine action of $\operatorname{Diff}\left(S^{1}\right)$ on $Q$.

Proposition (7.6) For the extension we have been studying the affine action of $\operatorname{Diff}\left(S^{1}\right)$ on $Q$ is given by

$$
\left(\phi^{-1}, q\right) \mapsto \phi^{*} q+\sigma(\phi),
$$

where if $q=a(\theta) d \theta^{2}$ then $\phi^{*} q=a(\phi(\theta)) \phi^{\prime}(\theta)^{2} d \theta^{2}$.

The proof of this is obvious from what has preceded. For an affine action is the same thing as a crossed homomorphism, and the crossed homomorphism $\sigma: \operatorname{Diff}\left(S^{1}\right) \rightarrow Q$ is determined by its derivative $D \sigma$ at the identity, which is given by

$$
D \sigma(\xi)=\frac{1}{12}\left(\xi^{\prime \prime \prime}+\xi^{\prime}\right)
$$

in agreement with the formula we found earlier for the cocycle defining the extension.

\section{Orbits}

According to Kirillov and Kostant's theory of "orbits" the irreducible unitary representations of a group $G$ are roughly in correspondence with a class of orbits of the action of $G$ on $\mathrm{g}^{*}$, the dual of the Lie algebra of $G$. More precisely, if $\alpha \in \mathrm{g}^{*}$ then the orbit $X_{\alpha}$ of $\alpha$ is naturally a symplectic manifold with a closed 2-form $\omega_{\alpha}$. The orbit is called integral if $\omega_{\alpha}$ defines an integral cohomology class in $H^{2}\left(X_{\alpha} ; \mathbb{R}\right)$. If $G_{\alpha}$ is the isotropy group of $\alpha$, and $\mathfrak{g}_{\alpha}$ is its Lie algebra, then $\alpha \mid \mathfrak{g}_{\alpha}$ is a homomorphism of Lie algebras $\mathfrak{g}_{\alpha} \rightarrow \mathbb{R}$, and one can ask whether it lifts to a character $\chi: G_{\alpha} \rightarrow \mathbb{T}$. If one is lucky then representations of $G$ will correspond to pairs $\left(X_{\alpha}, \chi\right)$, where $X_{\alpha}$ is an integral orbit and $\chi: G_{\alpha} \rightarrow \mathbb{T}$ is a lift of $\alpha \mid \mathfrak{g}_{\alpha}$.

It would be very optimistic to hope for such a correspondence to exist in the case of the infinite dimensional groups considered in this paper. Nevertheless it is interesting to inspect the orbits to see whether any of them show signs of corresponding to the representations we have found.

The groups we have considered are central extensions by $\mathbb{T}$ of $\Gamma=\operatorname{Map}\left(S^{1} ; G\right)$ and $\operatorname{Diff}\left(S^{1}\right)$, where $G$ is a compact group. Their Lie algebras can accordingly be identified with $\mathbb{R} \oplus \operatorname{Map}\left(S^{1} ; \mathfrak{g}\right)$ and $\mathbb{R} \oplus \operatorname{Vect}\left(S^{1}\right)$. The duals of $\operatorname{Map}\left(S^{1} ; \mathfrak{g}\right)$ and Vect $\left(S^{1}\right)$ are spaces of distributions on the circle; but we shall consider here only the "smooth" part of the duals, identified respectively with $L=\operatorname{Map}\left(S^{1} ; \mathrm{g}\right)$ (using an invariant inner product on $\mathfrak{g}$ ) and $Q$, the space of quadratic differentials on $S^{1}$. Thus we have to consider the action of $\Gamma$ on $\mathbb{R} \oplus L$ and the action of $\operatorname{Diff}\left(S^{1}\right)$ on $\mathbb{R} \oplus Q$. As we are interested only in representations which are faithful on the centre $\mathbb{T}$ we need only consider the orbits in $1 \oplus L$ and $1 \oplus Q$. It turns out in both cases that these orbits are very easy to classify (unlike the orbits in $0 \oplus L$ and $0 \oplus Q$ ).

(a) The Case of $\Gamma=\operatorname{Map}\left(S^{1} ; G\right)$, where $G$ is Compact

The action of $\Gamma$ on $1 \oplus L$ will be thought of as an affine action on $L$ :

$$
(f, \xi) \mapsto f * \xi=f \cdot \xi+a_{f},
$$


where $f \in \Gamma$ and $\xi \in L$, and $f \cdot \xi$ denotes the obvious adjoint action of $f$ on $\xi$. Because $f \mapsto a_{f}$ is a crossed homomorphism it is determined by its derivative at the identity element, which is $\eta \mapsto \omega(\eta, \cdot)$, where $\omega: L \times L \rightarrow \mathbb{R}$ is the Lie algebra cocycle defining the extension $\hat{\Gamma}$ of $\Gamma$. Recalling that

$$
\omega(\xi, \eta)=\frac{\pi}{2 \pi} \int_{0}^{2 \pi}\left\langle\xi^{\prime}(\theta), \eta(\theta)\right\rangle d \theta
$$

we find

Proposition (8.1) $a_{f}(\theta)=f^{\prime}(\theta) f(\theta)^{-1}$

Note. Here, and from now on, I shall employ notation as if $G$ were a matrix group, and $f: S^{1} \rightarrow G$ a matrix-valued function. Thus the adjoint action of $f$ on $\xi$ above is given by $(f \cdot \xi)(\theta)=f(\theta) \xi(\theta) f(\theta)^{-1}$.

For any $\xi \in L$ there is a unique function $F_{\xi}:[0,2 \pi] \rightarrow G$ such that $F_{\xi}(0)=1$ and $F_{\xi}^{\prime}(\theta)=\xi(\theta) F_{\xi}(\theta)$ for $0 \leqq \theta \leqq 2 \pi$. Let us define $g: L \rightarrow G$ by $g(\xi)=F_{\xi}(2 \pi)^{\xi}$.

Proposition (8.2) (i) $g(f * \xi)=f(0) g(\xi) f(0)^{-1}$,

(ii) the orbits of the affine action of $\Gamma$ on $L$ are the inverse-images by $g: L \rightarrow G$ of the conjugacy classes of $G$,

(iii) each orbit contains a constant map $\xi: S^{1} \rightarrow \mathfrak{g}$, and

(iv) the isotropy group of any $\xi \in L$ is isomorphic (via $f \mapsto f(0)$ ) to the centralizer of $g(\xi)$ in $G$.

Proof. (i) Because the solutions of ordinary differential equations are unique one has $F_{f * \xi}(\theta)=f(\theta) F_{\xi}(\theta) f(0)^{-1}$.

(ii) If $g(\xi)=g(\eta)$ then define $f \in \Gamma$ by $f(\theta)=F_{\eta}(\theta) F_{\xi}(\theta)^{-1}$. (This does belong to $\Gamma$, being smooth at $\theta=0=2 \pi$.) By calculation it appears that $f * \xi=\eta$.

(iii) Given any $\xi \in L$ choose $\xi_{0} \in \mathfrak{g}$ so that $\exp \left(2 \pi \xi_{0}\right)=g(\xi)$. Regarding $\xi_{0}$ as a constant element of $L$ we have $F_{\xi_{0}}(\theta)=\exp \left(\theta \xi_{0}\right)$ and so $g\left(\xi_{0}\right)=g(\xi)$.

(iv) We have $f * \xi=\xi$ if and only if

$$
f^{\prime}(\theta)=[\xi(\theta), f(\theta)] .
$$

This equation is uniquely soluble for $f$ given $f(0)$ providing $f(0)$ commutes with $g(\xi)$. (It is enough to consider the case when $\xi$ is constant: then the solution is $f(\theta)=e^{\theta \xi} f(0) e^{-\theta \xi}$.)

Now let us consider some constant $\xi \in \mathfrak{g} \subset L$ and ask whether $\langle\xi$,$\rangle lifts to a$ character of its isotropy group, the centralizer $Z$ of $\exp (2 \pi \xi)$ in $G$. Let $T$ be a maximal torus of $G$ whose Lie algebra contains $\xi$. It is well-known that if $\langle\xi$, $\rangle$ is a weight of $T$ then $\exp (2 \pi \xi)$ is in the centre of $G$, so that $Z=G$. On the other hand if $\exp (2 \pi \xi)$ is in the centre of $G$ then it is easy to see that $\langle\xi$,$\rangle is liftable. The orbit of$ $0 \in \mathfrak{g}$ is $\Gamma / G \cong \Omega G$, the loop-space of $G$, and one can identify it with a subgroup of $\Gamma$. It is easy to check that its symplectic form is the 2-form discussed in Sect. 7, the Chern class of the central extension regarded as a circle-bundle on $\Omega G$. The other orbits can be identified with the connected components of $\Omega G^{\prime}$, where $G^{\prime}$ is the quotient of $G$ by its centre.

We have seen in Sect. 4 that the representations of $\Gamma$ of positive energy are indexed by the centre of $G$, and Kač's method leads to the same conclusion. So the 
suggestion of the orbit method would be that all the projective unitary representations with the cocycle we are considering are of positive energy.

(b) $\operatorname{Diff}\left(S^{1}\right)$ and Hill's Equations

The smooth part of the dual of the Lie algebra of the central extension of $\operatorname{Diff}\left(S^{1}\right)$ that we are concerned with can be identified with $R \oplus Q$, where $Q$ is the space of quadratic differentials on $S^{1}$, and the projective representations of $\operatorname{Diff}\left(S^{1}\right)$ with the given cocycle ought to correspond to the orbits of $\operatorname{Diff}\left(S^{1}\right)$ on $1 \oplus Q \cong Q$. We have seen in Sect. 7 that the action is the affine action

$$
\left(\phi^{-1}, q\right) \mapsto \phi^{*} q+\sigma(\phi)
$$

where $\sigma(\phi)$ is the Schwarzian derivative.

The space $1 \oplus Q$ has an interesting interpretation in terms of Hill's equations. A Hill's operator is a second-order linear differential operator on the circle of the form

$$
D_{q}=\left(\frac{d}{d \theta}\right)^{2}+q(\theta),
$$

where $q$ is a real-valued function on $S^{1}$. If the parameter $\theta$ is changed by a diffeomorphism $\theta \mapsto \phi(\theta)$ so that $d / d \theta$ becomes $\left(\phi^{\prime}\right)^{-1} d / d \theta$ then $D_{q}$ becomes

$$
\frac{1}{\left(\phi^{\prime}\right)^{2}}\left(\frac{d}{d \theta}\right)^{2}-\frac{\phi^{\prime \prime}}{\left(\phi^{\prime}\right)^{3}} \frac{d}{d \theta}+q(\phi(\theta)) .
$$

It is very easy to check that this is

$$
M\left(\left(\phi^{\prime}\right)^{3 / 2}\right) \cdot D_{q} \cdot M\left(\left(\phi^{\prime}\right)^{-1 / 2}\right),
$$

where $\tilde{q}(\theta)=q(\phi(\theta)) \cdot \phi^{\prime}(\theta)^{2}+S(\phi)$, and $M(\psi)$ is the operation of multiplication by $\psi$. This means that if $D_{q}$ is regarded not as an operator on functions on $S^{1}$ but as an operator taking of weight $\frac{1}{2}$ to densities of weight $\frac{3}{2}$ then the natural transform of $D_{q}$ by $\phi$ is $D_{\tilde{q}}$. We have, therefore, a natural action of $\operatorname{Diff}\left(S^{1}\right)$ on the space of Hill's operators, and can identify the Hill's operators with the affine space $1 \oplus Q$. To make the fit precise we shall redefine $D_{q}$ as $(d / d \theta)^{2}+\hat{q}$, where $\hat{q}=6 q+\frac{1}{4}$.

The orbits of this action have been studied in [9]. I shall recall the result here, as I wish to state it differently (and the result in [9] seems not quite correct). Consider the equation $D_{q} f=0$ as an equation on $\mathbb{R}$ rather than $S^{1}$. Let $V_{q}$ be its twodimensional space of solutions. Evaluation at a point $\theta \in \mathbb{R}$ defines an element of the dual space $V_{q}^{*}$, or, more accurately, of the projective line $P\left(V_{q}^{*}\right)$, as $V_{q}$ consists of densities rather than functions. Thus to $D_{q}$ is associated a natural map $F_{q}: \mathbb{R} \rightarrow P\left(V_{q}^{*}\right)$, which is easily seen to be a local homeomorphism. Because $D_{q}$ has periodic coefficients the operation of translation by $2 \pi$ induces a map $M_{q}: V_{q} \rightarrow V_{q}$ called the monodromy of $D_{q}$, such that the following diagram commutes:

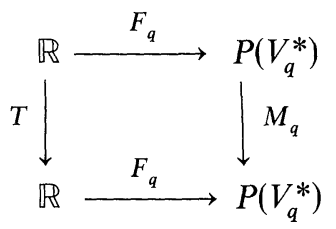


where $T$ is $\theta \mapsto \theta+2 \pi$. Conversely if one is given a smooth immersion $F: \mathbb{R} \rightarrow P_{\mathbb{R}}^{1}$ and a projective automorphism $M$ of $P_{\mathbb{R}}^{1}$ such that $M F=F T$ then it is clear that the pair $(F, M)$ arises from a unique Hill's equation. So Hill's equations can be identified with pairs $(F, M)$, where two such pairs are regarded as the same if they differ by an automorphism of $P_{\mathbb{R}}^{1}$. The action of a diffeomorphism $\phi$ of $S^{1}$ on $(F, M)$ simply replaces it by $(F \tilde{\phi}, M)$, were $\tilde{\phi}: \mathbb{R} \rightarrow \mathbb{R}$ is a lift of $\phi$. Now for a given operator $D_{q}$ the monodromy $M_{q}$ determines a well-defined conjugacy class in $\mathrm{PGL}_{2}(\mathbb{R})$; but (because $\left.\pi_{1} \mathrm{PGL}_{2}(\mathbb{R}) \cong \pi_{1} P_{\mathbb{R}}^{1}\right)$ the path $F_{q}$ determines a definite lift of this to a conjugacy class in $S L_{2}(\mathbb{R})$, the simply-connected covering group of $\mathrm{PGL}_{2}(\mathbb{R})$. The following result is now obvious:

Proposition (8.3) The orbits of $\operatorname{Diff}\left(S^{1}\right)$ on $1 \oplus Q$ correspond precisely (by assigning to $D_{q}$ its lifted monodromy) to the conjugacy classes of $\widetilde{S L}_{2}(\mathbb{R})$.

Note. $S L_{2}(\mathbb{R})$ has an outer involution, and the preceding statement really refers to conjugacy under the full automorphism group.

The conjugacy classes in $S L_{2}(\mathbb{R})$ are determined by the trace, and are of three types, elliptic, parabolic and hyperbolic. The conjugacy classes in $\widetilde{S L}_{2}(\mathbb{R})$ are correspondingly of three types. The elliptic ones are all on one-parameter subgroups, and are determined by giving, up to sign, a number $\theta \in \mathbb{R}$ such that $2 \cos \theta=$ (trace). The parabolic and hyperbolic classes from a disconnected space with components corresponding to $\mathbb{Z}$ : they are determined by their trace and the number of their component - evidently only those in the 0 -component lie on one-parameter subgroups.

When the lifted monodromy lies on a one-parameter subgroup (and not otherwise) the corresponding orbit contains a representative with $q$ constant. (The other orbits can presumably be represented by Mathieu equations, but the results of [9] seem wrong at this point.) For these orbits the isotropy group always contains the group $R$ of rotations of the circle. It is possible to lift $q$ to a character of $R$ only if $q$ is an integer. The monodromy $M_{q}$ is

$$
\left(\begin{array}{lr}
\cos 2 \pi \sqrt{ } q & -\sin 2 \pi \sqrt{ } q \\
\sin 2 \pi \sqrt{ } q & \cos 2 \pi \sqrt{ } q
\end{array}\right)
$$

in $\mathrm{PGL}_{2}(\mathbb{R})$ if $q \geqq 0$ (and an analogous hyperbolic element if $q<0$ ). This is the identity if $\hat{q}=\frac{1}{4} m^{2}$, i.e. $q=\frac{1}{24}\left(m^{2}-1\right)$, where $m$ is an integer. The isotropy group of $q$ is $R$ if $\hat{q} \neq \frac{1}{4} m^{2}$, but if $\hat{q}=\frac{1}{4} m^{2}$ it jumps in dimension from 1 to 3 , and becomes the $m$-fold covering of $\mathrm{PGL}_{2}(\mathbb{R})$ consisting of the elements of $\operatorname{Diff}\left(S^{1}\right)$ which are periodic with period $2 \pi / m$ and are $m$-fold coverings of diffeomorphisms in $\mathrm{PGL}_{2}(\mathbb{R})$.

All this means that the elliptic orbits correspond rather well in a qualitative way with the results of Sect. 6 , and with Kač's results, but the values of $q$ predicted are not quite right, as often happens with the orbit method.

\section{Appendix}

A string is the image of a smooth map $x:[0,1] \rightarrow \mathbb{R}^{3}$. When it moves it sweeps out a "world-surface" $x:[0,1] \times \mathbb{R} \rightarrow \mathbb{R}^{4}$ in Minkowski space-time (I shall take the metric in Minkowski space to be $\left.-d t^{2}+d x^{2}+d y^{2}+d z^{2}\right)$. The particles of which 
the string is made up are regarded as indistinguishable, so the parametrization has no physical significance. Nevertheless I shall suppose that the string can be parametrized in such a way that its individual particles move no faster than light, i.e. $\left\langle\frac{\partial x}{\partial t}, \frac{\partial x}{\partial t}\right\rangle \leqq 0$. Then the world-surface between times $t_{0}$ and $t_{1}$ has an area

$$
\int_{t_{0}}^{t_{1}} d t \int_{0}^{1}\left\{\left\langle\frac{\partial x}{\partial s}, \frac{\partial x}{\partial t}\right\rangle^{2}-\left\langle\frac{\partial x}{\partial s}, \frac{\partial x}{\partial s}\right\rangle\left\langle\frac{\partial x}{\partial t}, \frac{\partial x}{\partial t}\right\rangle\right\}^{1 / 2} d s
$$

The motion of the string is supposed to be governed by an action-principle which requires this area to be minimized, or at any rate to be stationary.

If the world-surface lay in Euclidean rather than Minkowski space this would not make sense, as we should have a soap-film without a wire, and the string would collapse completely. But in Minkowski space the problem is well-posed, and we see that the ends of the string must move with the velocity of light at right angles to the string, for then and only then will the area of the surface not change to first order if one simply shrinks the string in on itself.

I shall make two more assumptions. The first is that the motion is such that if one sends a light signal along the string from either end then it will get to the other end in a finite time. That seems reasonable physically, as the part of the string reachable by light signals from one end will presumably behave independently of the rest. Granting this assumption there is a natural parametrization of the world-surface which assigns to a point $P$ the pair $\left(t, t^{\prime}\right)$, where $t$ is the time at which one must send a light-signal from the end along the string to arrive at $P$, at $t^{\prime}$ is the time at which a signal from $P$ will reach the end. (I am choosing a preferred end of the string.)

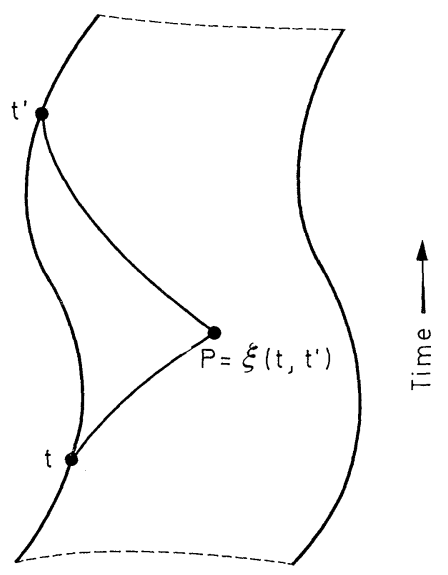

Fig. 2

This parametrization is a map

$$
\xi:\left\{\left(t, t^{\prime}\right) \in \mathbb{R}^{2}: t \leqq t^{\prime} \leqq t+2 \ell(t)\right\} \rightarrow \mathbb{R}^{4},
$$

where $2 \ell(t)$ is the time taken for a signal to travel from the preferred end of the string to the other end and back, beginning at time $t$. 
The second assumption is more technical. Suppose that for a point $x$ near the edge of the world-surface the times taken for forwards and backwards light signals from $x$ to reach the edge are $t_{+}(x)$ and $t_{-}(x)$. I shall suppose that the ratio $t_{+}(x) / t_{-}(x)$ tends to 1 as $x$ approaches the edge of the surface. (I think that this is equivalent to assuming that the string does not curl up at its ends, i.e. that its curvature is bounded at any time.) Then we have

Proposition (A.1) (a) $\ell(t)=\ell$ is independent of $t$, and

(b) $\xi\left(t, t^{\prime}\right)=\frac{1}{2}\left(f(t)+f\left(t^{\prime}\right)\right)$, where $f: \mathbb{R} \rightarrow \mathbb{R}^{4}$ satisfies

(i) $f(t+2 \ell)-f(t)=2 \pi p$ is independent of $t$, and

(ii) $\left\langle f^{\prime}(t), f^{\prime}(t)\right\rangle=0$.

It is easy to check that $p$ is the total momentum of the string.

The map $f: \mathbb{R} \rightarrow \mathbb{R}^{4}$ is the trajectory of the end of the string parametrized by the time coordinate in the particular Lorentz frame. It is more natural to reparametrize it invariantly by a parameter $\theta$ so that

$$
\left.\begin{array}{l}
\left\langle f^{\prime}(\theta), p\right\rangle=\langle p, p\rangle \text { and } \\
f(\theta+2 \pi)=f(\theta)+2 \pi p .
\end{array}\right\}
$$

These requirements fix the parametrization up to an additive constant.

The significance of Proposition (A.1) is that the motions of the string are completely equivalent to those of a point particle which moves with the velocity of light along a trajectory $f: \mathbb{R} \rightarrow \mathbb{R}^{4}$ satisfying $\left(^{*}\right)$. The particle has a well-defined rest-frame in which it describes a periodic closed orbit with the speed of light.

\section{Proof of Proposition (A.1)}

If the world-surface is parametrized arbitrarily in terms of parameters $(u, v)$ then the Euler-Larange equations for the variational problem are

$\frac{\partial}{\partial u}\left(\frac{1}{\sqrt{F}}\left(\left\langle x_{u}, x_{v}\right\rangle x_{v}-\left\langle x_{v}, x_{v}\right\rangle x_{u}\right)\right)+\frac{\partial}{\partial v}\left(\frac{1}{\sqrt{F}}\left(\left\langle x_{u}, x_{v}\right\rangle x_{u}-\left\langle x_{u}, x_{v}\right\rangle x_{v}\right)\right)=0$, where $F=\left\langle x_{u}, x_{v}\right\rangle^{2}-\left\langle x_{u}, x_{u}\right\rangle\left\langle x_{v}, x_{v}\right\rangle$, and $x_{u}=\partial x / \partial u, x_{v}=\partial x / \partial v$. If the parametrization is such that $x_{u}$ and $x_{v}$ are light-like then the equations simplify to

$$
\frac{\partial^{2} x}{\partial u \partial v}=0
$$

That is the case for the preferred parametrization introduced above, so we find

$$
\xi\left(t, t^{\prime}\right)=f_{1}(t)+f_{2}\left(t^{\prime}\right),
$$

for some functions $f_{1}, f_{2}: \mathbb{R} \rightarrow \mathbb{R}^{4}$ such that $f_{1}^{\prime}(t)$ and $f_{2}^{\prime}\left(t^{\prime}\right)$ are light-like. The trajectory of one end of the string is $t \mapsto f_{1}(t)+f_{2}(t)$, and so $f_{1}^{\prime}(t)+f_{2}^{\prime}(t)$ must be light-like too. This means that $f_{1}^{\prime}(t)$ is parallel to $f_{2}^{\prime}(t)$.

Now we use the second assumption. Let $f_{i}^{0}$ denote the time component of $f_{1}$. The time-coordinate of $\xi\left(t, t^{\prime}\right)$ is $f_{i}^{0}(t)+f_{2}^{0}\left(t^{\prime}\right)$, and the times taken for forward and backward signals from $\xi\left(t, t^{\prime}\right)$ to the end are $t^{\prime}-f_{1}^{0}(t)-f_{2}^{0}(t)$ and $f_{1}^{0}(t)+f_{2}^{0}(t)-t$, 
i.e. $f_{1}^{0}\left(t^{\prime}\right)-f_{1}^{0}(t)$ and $f_{2}^{0}\left(t^{\prime}\right)-f_{2}^{0}(t)$. If the ratio of these tends to 1 as $t^{\prime} \rightarrow t$ then $f_{1}^{0^{\prime}}(t)=f_{2}^{0^{\prime}}(t)$, and so $f_{1}^{\prime}=f_{2}^{\prime}$, and we can suppose $f_{1}=f_{2}=\frac{1}{2} f$.

The trajectory of the other end of the string is $t \mapsto \frac{1}{2}(f(t-\ell(t))+f(t+\ell(t)))$. For this to be light-like $f^{\prime}(t-\ell(t))$ and $f^{\prime}(t+\ell(t))$ must be parallel. But the time-component of each is 1 , so $f^{\prime}(t-\ell(t))=f^{\prime}(t+\ell(t))$.

Finally we apply the second assumption at this end. Suppose that $t^{\prime}$ is slightly greater than $t$. The point $\left.\xi\left(t^{\prime}, t+2 \ell\right)\right)$ has time-coordinate $\frac{1}{2}\left(t+t^{\prime}\right)+\ell(t)$, and signals from it research the end at $t+\ell(t)$ and $t^{\prime}+\ell\left(t^{\prime}\right)$. From this we find that $\left(\frac{1}{2}\left(t^{\prime}-t\right)+\ell\left(t^{\prime}\right)-\ell(t)\right) / \frac{1}{2}\left(t^{\prime}-t\right) \rightarrow 1$ as $t^{\prime} \rightarrow t$, and so that $\ell^{\prime}(t)=0$. Thus $\ell(t)$ is constant, and $f^{\prime}(t+2 \ell)=f^{\prime}(t)$. This gives the desired result $f(t+2 \ell)=f(t)+2 \pi p$ for some vector $p$.

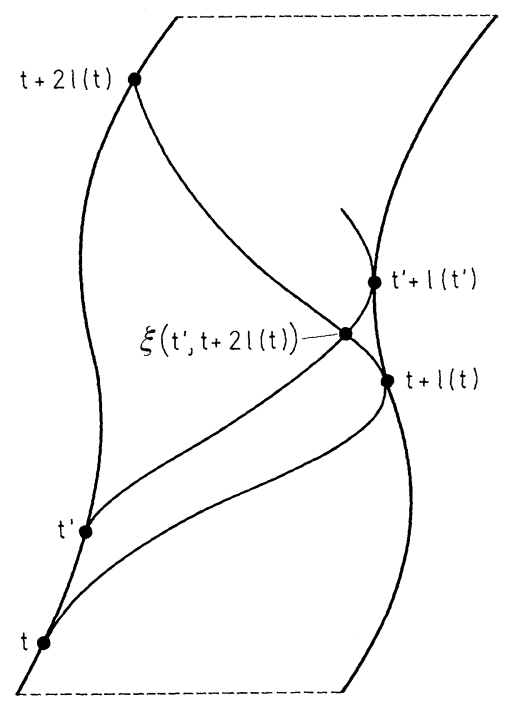

Fig. 3

Before turning to the quantization of the system I shall make a few general remarks.

Suppose that $Y$ is a symplectic manifold on which a group $G$ acts, preserving the symplectic form. Then there is defined a natural map, the "momentum" map, $P: Y \rightarrow \mathfrak{g}^{*}$, where $\mathrm{g}$ is the Lie algebra of $G$, and $\mathrm{g}^{*}$ is its dual. $P$ is simply the transpose of the map $\mathfrak{g} \rightarrow \operatorname{Map}(Y ; \mathbb{R})$ which assigns to an element of $\mathfrak{g}$ a Hamiltonian function for the corresponding flow on $Y$. (The Hamiltonian are determined only up to an additive scalar, and $P$ is more properly a map $Y \rightarrow \hat{\mathfrak{g}}^{*}$, where $\hat{\mathrm{g}}$ is the central extension of $\mathfrak{g}$ by $\mathbb{R}$ determined by $Y$.) Clearly $P$ is equivariant with respect to $G$. If one chooses an orbit $\omega$ of the coadjoint action of $G$ on $*$ then $Y_{\omega}=P^{-1}(\omega)$ is $G$-invariant, and $X_{\omega}=Y_{\omega} / G$ has, if it is a manifold, a natural symplectic structure. $X_{\omega}$ is called the quotient system of $Y$ by $G$ with momentum $\omega$. If $\omega_{0}$ is a point of the orbit $\omega$ notice that $X_{\omega}$ can be identified with $X_{\omega_{0}}=Y_{\omega_{0}} / G_{0}$, where $Y_{\omega_{0}}=P^{-1}\left(\omega_{0}\right)$, and $G_{0}$ is the stabilizer of $\omega_{0}$.

The state space $X$ of a string is a quotient system of this form. Suppose that $Y$ is 
the space of all smooth maps $f: \mathbb{R} \rightarrow \mathbb{R}^{4}$ such that

$$
P_{f}=\frac{1}{2 \pi}(f(\theta+2 \pi)-f(\theta))
$$

is constant. $Y$ is a vector space, and has a symplectic structure given by the skew form $S: Y \times Y \rightarrow \mathbb{R}$ defined by

$$
S\left(f_{1}, f_{2}\right)=\frac{1}{4 \pi} \int_{0}^{2 \pi}\left(\left\langle f_{1}, f_{2}^{\prime}\right\rangle-\left\langle f_{1}^{\prime}, f_{2}\right\rangle\right) d \theta+\frac{1}{2}\left(\left\langle f_{1}(0), p_{f_{2}}\right\rangle-\left\langle p_{f_{1}}, f_{2}(0)\right\rangle\right) .
$$

Let $G$ denote the universal covering group of $\operatorname{Diff}\left(S^{1}\right)$, the space of diffeomorphisms $\phi: \mathbb{R} \rightarrow \mathbb{R}$ such that $\phi(\theta+2 \pi)=\phi(\theta)+2 \pi$. $G$ acts on $Y$ preserving $S$, and the Hamiltonian function $H_{\xi}$ inducing the action of the vector field $\xi(\theta) d / d \theta$ is given by

$$
H_{\xi}(f)=S\left(\xi f^{\prime}, f\right)=\frac{1}{2 \pi} \int_{0}^{2 \pi} \xi(\theta)\left\langle f^{\prime}(\theta), f^{\prime}(\theta)\right\rangle d \theta .
$$

In other words, the momentum map $P: Y \rightarrow \mathfrak{g}^{*}$ is $f \mapsto\left\langle f^{\prime}, f^{\prime}\right\rangle$.

This means that $Y_{0}=P^{-1}(0)$ consists of the trajectories $f \in Y$ which travel with the speed of light. Each such has a unique preferred parametrization, and so $X_{0}=Y_{0} / G$ is precisely the state-space $X$ of a string.

In favorable cases quotient systems of the form $X_{\omega}$ can be quantized as follows. One first chooses a quantization of $Y$, a certain Hilbert space $H$. The action of $G$ on $Y$ corresponds to a unitary action of $G$ on $H$. This can be decomposed as a direct integral

$$
H=\int H_{\omega} \otimes P_{\omega},
$$

where $P_{\omega}$ runs through the irreducible unitary representations of $G$. If the irreducible representations $P_{\omega}$ can be indexed by the orbits $\omega$ of $G$ in $g^{*}$ then the Hilbert space $H_{\omega}$ ought to be the quantization of the quotient system $X_{\omega}$.

When one attempts to apply this ideal procedure to the states of a string at least two difficulties arise. The first appears when one quantizes any infinite dimensional linear system $Y$ on which a group $G$ acts: the extension of $\mathfrak{g}$ by $\mathbb{R}$ defined by $Y$ is trivial, as each $\xi \in \mathfrak{g}$ has a canonical Hamiltonian which is a homogeneous quadratic function on $Y$; on the other hand when $Y$ is quantized in the standard "metaplectic" way a non-trivial central extension $\hat{G}$ of $G$ acts on $H$, and the representations of $\hat{G}$ which occur in $H$ correspond, if they correspond to orbits at all, to orbits in a certain affine action of $G$ on $\mathrm{g}^{*}$ (cf. Sect. 8). It is thus not clear which $H_{\omega}$ to associate to which values of the classical momentum.

The second difficulty is that one cannot quantize the linear system $Y$ satisfactorily. For to do so we should presumably begin by observing that the $\mathbb{R}^{4}$ valued function $f \mapsto p_{f}$ on $Y$ corresponds to the total momentum of the system, and the corresponding operator (actually four commuting operators) should break up $H$ as

$$
\int_{p \in \mathbb{R}^{4}} H_{p}
$$

where $H_{p}$ is got by quantizing the linear system $V_{p}=\left\{f \in Y: p_{f}=p\right\} / \mathbb{R}^{4}$. (Thus 
$V_{p}$ is a quotient system of $Y$ by the group $\mathbb{R}^{4}$ of translations.) To quantize $V_{p}$ one would choose a positive polarization $V_{p, \mathbb{C}}=W \oplus \bar{W}$ and define $H_{p}=\hat{S}(W)$, as the body of this paper. But unfortunately, because the inner product in Minkowski space is indefinite one cannot choose the polarization both positive and invariant under the Lorentz group. The standard procedure is to choose an invariant polarization $W$ which is not positive (it is the part of $V_{p, \mathbb{C}}$ of "positive energy" in the sense of this paper), and to form a pseudo-Hilbert-space $H_{p}=\hat{S}(W)$ which has an indefinite inner product. The group $G$ acts projectively on $H_{p}$, and it has a discrete decomposition

$$
H_{p}=\underset{n \geqq 0}{\bigoplus} H_{p}^{\left(\lambda_{n}\right)} \oplus P_{\lambda_{n}},
$$

where $P_{\lambda_{n}}$ is the irreducible representation of $G$ with lowest weight $\lambda_{n}=n+\frac{1}{2}\langle p, p\rangle$ which need not be integral here as $G$ is now not $\operatorname{Diff}\left(S^{1}\right)$ but its covering group.

One wants to pick out in $H_{p}$ the isotypical piece $H_{p}^{(\alpha)}$ associated to a certain irreducible representation $P_{\alpha}$ of $G$ taken to correspond to the momentum condition $\left\langle f^{\prime}, f^{\prime}\right\rangle=0$. This will be zero unless $\frac{1}{2}\langle p, p\rangle-\alpha$ is a negative integer. Physicists customarily take $\alpha=1$, for a reason I shall explain in a moment, though this has the great disadvantage that the Hilbert space then contains state vectors with $\langle p, p\rangle=2$, i.e. particles moving faster than light with imaginary mass, so called "tachyons". The general nature of the model, on the other hand, leads one to suppose that the lowest state of a string ought to be when it collapses to a particle moving with the speed of light: that would correspond to $\alpha=0$.

Goddard and Thorn [4] have shown that when $\alpha=1$ the metric of $H_{p}^{(\alpha)}$ is positive semi-definite. By dividing $H_{p}^{(\alpha)}$ by its radical one obtains a genuine Hilbert space $\tilde{H}_{p}^{(\alpha)}$, and physicists take

$$
\tilde{H}=\int \tilde{H}_{p}^{(\alpha)},
$$

where $p$ runs over all momentum vectors in $\mathbb{R}^{4}$ such that $\frac{1}{2}\langle p, p\rangle+\alpha$ is a negative integer, as the Hilbert space of quantum states of the string. (I should perhaps mention that physicists define $H_{p}^{(\alpha)}$ as the subspace $\left\{\xi \in H_{p}: V_{0} \xi=\alpha \xi, V_{-k} \xi=0\right.$ for $k>0\}$ of $H_{p}$ (where the $V_{k}$ are the basis for $\mathscr{V}$ ), exploiting the fact that each irreducible representation of $G$ contains a unique lowest-weight vector.)

To produce a space of states of a free string is not of interest unless one can describe how strings interact. I am certainly not competent to do that, but I shall simply point out, as it was the motivation for Sect. 4 of this paper, that one can define for certain vectors $v \in \mathbb{R}^{4}$ an operator $B^{v}: H_{p} \rightarrow H_{p+v}$ which commutes with the action of $G$ and is supposed to correspond to the process of absorption of a particle of momentum $v$ by a string of momentum $p$ to form a string of momentum $p+v$.

One wants $B_{v}$ to be defined only when $\frac{1}{2}\langle p+v, p+v\rangle-\frac{1}{2}\langle p, p\rangle$, i.e. $\langle p, v\rangle+\frac{1}{2}\langle v, v\rangle$, is integral. Now the elements $f$ of the additive group $Y$ described above act projectively on $H$ by operators $A(f)$. If $\langle v, v\rangle=2$, and we restrict ourselves to the $H_{p}$ such that $\langle p, v\rangle$ is integral, then just as in the last part of Sect. 4 we can define for each $\theta \in \mathbb{R}$ a "blip" $B_{\theta}^{v}: H_{p} \rightarrow H_{p+v}$. The integral

$$
B^{v}=\frac{1}{2 \pi} \int_{0}^{2 \pi} B_{\theta}^{v} d \theta
$$


is an operator which commutes with $G$, and is what we want. But it makes sense only when $\langle v, v\rangle=2$, and the particle absorbed is a tachyon. Physicists want the particles absorbed to correspond to unexcited states of a string, i.e. to those such that $\frac{1}{2}\langle p, p\rangle-\alpha=0$, and they are therefore led to assume that $\alpha=1$.

\section{References}

0. Frenkel, I. B., Kač, V. G. : Basic representations of affine Lie algebras and dual resonance models. Inventiones math. 62, 23-66 (1980)

1. Gel' fand, I. M., Vilenkin, N. Ya. : Generalized Functions, Vol. 4. New York: Academic Press 1964

2. Gel'fand, I. M., Fuks, D. B. : Funkt. Anal. Jego Prilozh. 2, 92-93 (1968)

3. Goddard, P., Horsley, R. : Nucl. Phys. B111, 272-296 (1976)

4. Goddard, P., Thorn, C. B. : Phys. Lett. 40B, 235-238 (1972)

5. Jacob, M. (ed.): Dual theory. Amsterdam: North-Holland 1974

6. Kač, V. G. : Adv. Math. 30, 85-136 (1978)

7. Kač, V. G. : Contravariant form for infinite- dimensional Lie algebras and superalgebras. (to apper)

7a. Kač, V. G. : Adv. Math. 35, 264-273 (1980)

8. Kirillov, A. A. : Unitary representations of the group of diffeomorphisms of a manifold. (to appear)

9. Lazutkin, V. F., Pankratova, T. F. : Funkt. Anal. Jego Prilozh. 9, 41-48 (1975) (Russian)

10. Shale, D. : Trans. Am. Math. Soc. 103 149-167 (1962)

11. Shale, D. : Stinespring, W. F. : J. Math. Mech. 14, 315-322 (1965)

12. Vergne, M. : Seconde quantification et groupe symplectique. C. R. Acad. Sci. (Paris), 285, A 191-194 (1977)

13. Vershik, A. M., Gel'fand, I. M., Graev, M. I. : Usp. Mat. Nauk 30, 1-50 (1975)

14. Vershik, A. M., Gel'fand, I. M., Graev, M. I. Dokl. Akad. Nauk. SSSR 232, 745-748 (1977)

Communicated by A. Jaffe

Received June 17, 1980; in revised form October 14, 1980 